\title{
Stochastic curvature flows: asymptotic derivation, level set formulation and numerical experiments
}

\author{
Markos A. KATSOUlaKis ${ }^{\dagger}$ AND Alvin T. KHO ${ }^{\ddagger \S}$ \\ Department of Mathematics and Statistics, University of Massachusetts, Amherst, \\ MA 01003-3110, USA
}

[Received 16 February 2000 and in revised form 2 October 2000]

\begin{abstract}
We study the effects of random fluctuations included in microscopic models for phase transitions on macroscopic interface flows. We first derive asymptotically a stochastic mean curvature evolution law from the stochastic Ginzburg-Landau model and develop a corresponding level set formulation. Secondly, we demonstrate numerically, using stochastic Ginzburg-Landau and Ising algorithms, that microscopic random perturbations resolve geometric and numerical instabilities in the corresponding deterministic flow.
\end{abstract}

Keywords: Stochastic Ginzburg-Landau models; Ising systems; stochastic curvature evolution; level sets; Monte Carlo methods.

\section{Introduction}

In this paper, we investigate the effects of microscopic random fluctuations on macroscopic models for phase transitions. Firstly, we study the manner in which such fluctuations-inherently present in microscopic models-are incorporated into the macroscopic description of interphase interface evolution. We formally show that under an appropriate scaling, the microscopic stochastic Ginzburg-Landau model yields a macroscopic front that moves with normal velocity depending on its curvature and a non-Gaussian random driving term supported on the front itself. We refer to this interface evolution as a stochastic curvature flow, and we develop a corresponding level set formulation; the principal difficulty here being that the standard projection of a white noise driving term in $\mathbb{R}^{d}$ onto any codimension-one subspace, and hence onto the interface, is not well defined.

Secondly, the effect of microscopic random fluctuations to mean curvature flow is studied using Monte Carlo algorithms based upon stochastic Ginzburg-Landau models as well as nonconservative Ising systems. Simulations with these fundamentally different types of algorithms demonstrate that random fluctuations resolve geometric singularities of the corresponding deterministic evolutions (e.g. mean curvature flow) such as interface fattening and eliminate numerical instabilities. Similar outcomes are obtained for the Mullins-Sekerka flow where a conservative Ising system is employed as a microscopic approximation of the macroscopic free boundary problem. We want to point out that we are not proposing either the stochastic GinzburgLandau or the Ising model as a numerical alternative to conventional PDE based methods (e.g.

\footnotetext{
†Email: markos@math.umass.edu

*Email: alvin_kho@hms.harvard.edu

${ }^{\S}$ Current address: Children’s Hospital Informatics Program, Harvard Medical School, 300 Longwood Avenue, Boston, MA 02115, USA.
} 
level set, phase field) since these microscopic schemes are computationally intensive. Rather, our intention is to explore, from a microscopic perspective, the manner in which the random term handles instabilities like interface fattening, and demonstrate numerically the resulting probabilistic selection mechanism between all possible evolutions in the event of non-uniqueness in the deterministic flow. Furthermore, this analysis may provide some intuition in the construction of meso- or macroscopic differential-equation-based numerical models which are better suited for computation while, at the same time, including a random term that resolves geometric instabilities of the interface flow.

The paper is organized as follows. Section 2 is an overview of microscopic and phenomenological models of phase transition, their interconnections and relevant recent results. In Section 3, we consider the formal asymptotic analysis of a stochastic Ginzburg-Landau model yielding a stochastic mean curvature flow in the limit. In Section 4, we develop a level set formulation for the stochastic mean curvature flow. Section 5 contains numerical experiments employing the stochastic Ginzburg-Landau model to illustrate the resolution of interface fattening and instability in mean curvature flows via microscopic random fluctuations. In Section 6, we discuss similar experiments using Ising models with non-conservative, as well as conservative dynamics. The Appendix contains the proof of the $\ell^{2}$-stability of the approximating scheme used in Section 5 for the stochastic Ginzburg-Landau equation.

\section{Models of phase transition}

Phase transition is a phenomenon that is commonly observed in nature whereby solid, liquid or gaseous homogeneous forms (called phases) exist as distinct parts in a heterogeneous system. An example from materials science is the presence of local regions with separate atomic arrangements in solid polycrystalline media at low temperatures: see Allen and Cahn [1]. Generally, distinct phase regions within a medium are not stationary. At the microscopic level, molecules near the antiphase boundary, or indeed anywhere in the medium, may be constantly altering their physical or thermodynamic states, whereas at the macroscopic level, distinct bulk regions of one phase may interact or change their shapes and volume in the medium. The transition region between different phases is known as the interphase interface, or simply the interface. More often than not, there is no visibly clear separation between different phase regions as a result of the aforementioned kinetics.

A typical mathematical formulation of a multi-phase problem involves the definition of an order parameter $u: \mathcal{O} \times\{t \geqslant 0\} \rightarrow \mathcal{S} \subset \mathbb{R}$, where $\mathcal{O}$ represents the underlying physical medium in $\mathbb{R}^{d}$. The value $u(r, t)$ indicates the phase of the medium at the point $r$ at time $t$. This order parameter satisfies a prescribed equation of state or is calculated through microscopic master equations. The problem of tracking the interfacial motion through time translates into investigating the solution(s) of this equation of state given an initial datum corresponding to the initial state of the medium. In this paper, we mainly focus on two-phase isothermal systems with a non-conserved order parameter and briefly discuss a conserved case in Section 6. There are two major approaches to modelling phase transitions: microscopic and macroscopic (phenomenological).

Microscopic models treat phase transition as a Markov process set on a hypercubic lattice $\mathbb{Z}^{d}$, $d \geqslant 2$, known as an interacting particle system. Particles reside in lattice sites with individual states in a set $\mathcal{S}$, corresponding to different phases, which are dynamically updated by the Markov process with state space $\mathcal{S}^{\mathbb{Z}^{d}}$. The basic idea of the microscopic approach is to introduce, a priori, a Hamiltonian energy and the corresponding probability (Gibbs) measure on the state space, which 
give rise to the configurations of the entire system at equilibrium. Dynamics are defined as an updating mechanism on the configuration space that keeps the Gibbs measure invariant [32].

Following Spohn [32], we distinguish between Ising spin models where $\mathcal{S}=\{-1,+1\}$ and stochastic Ginzburg-Landau models where order parameters take continuous values in $\mathbb{R}$. A prototypical spin system is the ferromagnetic Ising spin model on $\mathbb{Z}^{d}$ with two phases, \pm 1 coexisting at sufficiently low temperatures $\beta^{-1}>0$. Each lattice site $x$ has a spin value $\sigma(x) \in\{-1,+1\}$. A spin configuration, $\sigma \doteq\left\{\sigma(x): x \in \mathbb{Z}^{d}\right\}$ is an element of the configuration space $\{-1,+1\}^{\mathbb{Z}^{d}}$, and is updated by a sequence of stochastic spin flips. A spin flip at $x$ denotes the event when $\sigma(x)$ switches its parity. On a finite volume $\Lambda \subset \mathbb{Z}^{d}$ one defines an ad hoc Hamiltonian for a particular configuration $\sigma$ as

$$
H_{\Lambda}^{h}(\sigma)=\sum_{x, y \in \Lambda, x \neq y} J(x, y) \sigma(x) \sigma(y)+h \sum_{x \in \Lambda} \sigma(x),
$$

where the function $J=J(x, y)>0$ denotes the interaction potential between two lattice sites $x$ and $y$, and $h$ is an external field. The spin flip dynamics is specified by a rate

$$
\begin{gathered}
c(x, \sigma)=\Psi\left(-\beta\left(H_{\Lambda}^{h}\left(\sigma^{x}\right)-H_{\Lambda}^{h}(\sigma)\right)\right), \\
\sigma^{x}(z) \doteq \begin{cases}\sigma(z) & \text { if } z \neq x \\
-\sigma(z) & \text { if } z=x,\end{cases}
\end{gathered}
$$

where $\sigma^{x}$ is the configuration after a spin flip at $x$ and the function $\Psi>0$ satisfies the detailed balance condition,

$$
\Psi(\lambda)=\Psi(-\lambda) \exp (-\lambda), \quad \lambda \in \mathbb{R} .
$$

Typical choices of dynamics are $\Psi(\lambda)=\left(1+\mathrm{e}^{\lambda}\right)^{-1}$ (Glauber) and $\Psi(\lambda)=\mathrm{e}^{-\lambda^{+}}$(Metropolis). Dynamics which obey the detailed balance condition leave the underlying Gibbs measures

$$
\mu_{\Lambda, \beta, h}(\sigma)=Z_{\Lambda, \beta, h}^{-1} \mathrm{e}^{-\beta H_{\Lambda}^{h}(\sigma)} \prod_{x \in \Lambda} \mathrm{d} \sigma(x)
$$

invariant under the prescribed spin flips. Here $\prod_{x \in \Lambda} \mathrm{d} \sigma(x)$ is the Bernoulli measure on $\Lambda$. The constant $Z_{\Lambda, \beta, h}$ normalizes $\mu_{\Lambda, \beta, h}$ to a probability measure on the configuration space $\mathcal{S}^{\Lambda}$. For Ising models with long-range interactions $J_{\gamma}(x, y)=\gamma^{d} J(\gamma(x-y))$, where $\gamma^{-1}>0$ denotes the interaction range between particles, De Masi et al. [12] (for Glauber dynamics) and Katsoulakis and Souganidis [25] (for general dynamics) have shown that there exist mesoscopic space scalings such that the limiting evolution of the average magnetization $m^{\gamma}(r, t)=\mathrm{E} \sigma_{t}(\gamma x), r=\gamma x \in \mathbb{R}^{d}$ as $\gamma \downarrow 0$ solves the non-local equation,

$$
\begin{gathered}
m_{t}+\Phi(\beta(J * m))[m-\tanh \beta(J * m+h)]=0, \\
(r, t) \in \mathbb{R}^{d} \times\{t \geqslant 0\},
\end{gathered}
$$

where $\Phi(\lambda)=\Psi(-2 \lambda)\left(1+\mathrm{e}^{-2 \lambda}\right), \lambda \in \mathbb{R}$. Equation (2.5) is a local mean field or mesoscopic description of phase transitions which includes microscopic information: for example, the interaction potential and dynamics. Ising spin models with conservative (spin exchange) dynamics will be discussed in Section 7. 
In stochastic Ginzburg-Landau (SGL) models, the order parameter has a continuous range: for example, $\mathcal{S}=\mathbb{R}$. Here, the Hamiltonian is defined as

$$
H_{\Lambda}(u)=\sum_{\substack{x, y \in \Lambda, x \neq y}} \frac{1}{2}(u(x)-u(y))^{2}+\sum_{x \in \Lambda} F(u(x)),
$$

where $F: \mathbb{R} \rightarrow \mathbb{R}$ is a double-well potential function with stable global minima $h_{ \pm}$and an unstable local maximum $h_{0}$. The dynamics of $u$ is governed by a system of stochastic differential equations indexed by $x \in \Lambda$,

$$
\mathrm{d} u(x, t)-\left(\Delta_{1} u(x, t)+F_{u}(u(x, t))\right) \mathrm{d} t+\sqrt{\frac{2}{\beta}} \mathrm{d} w(x ; t)=0,
$$

where $\Delta_{1}$ denotes the discrete Laplacian operator with the distance between neighbouring lattice points set to one and $\{w(x ; t): x \in \Lambda\}$ are independent Brownian motions. The dynamics (2.7) has a unique invariant Gibbs measure

$$
\mu_{\Lambda, \beta}(u)=Z_{\Lambda, \beta}^{-1} \mathrm{e}^{-\beta H_{\Lambda}(u)} \prod_{x \in \Lambda} \mathrm{d} u(x)
$$

where the constant $Z_{\Lambda, \beta}$ normalizes $\mu_{\Lambda, \beta}$ to a probability measure. The formal space continuum limit of (2.7), i.e. taking the distance between lattice points to zeros, yield

$$
\begin{gathered}
\frac{\partial}{\partial t} u(r, t)-\Delta u(r, t)+F_{u}(u(r, t))+\sqrt{\frac{2}{\beta}} \frac{\partial}{\partial r_{1}} \cdots \frac{\partial}{\partial r_{d}} \frac{\partial}{\partial t} W(r, t)=0, \\
(r, t) \in \mathbb{R}^{d} \times\{t \geqslant 0\},
\end{gathered}
$$

where $W(r, t)$ is a Brownian sheet. To simplify notation, we define

$$
J(r, t) \doteq \frac{\partial}{\partial r_{1}} \cdots \frac{\partial}{\partial r_{d}} \frac{\partial}{\partial t} W\left(r_{1}, \ldots, r_{d}, t\right)
$$

and refer to (2.10) as a white noise. See Walsh [34] for definitions and properties of the white noise. The continuous Hamiltonian that is associated with (2.9) is the Ginzburg-Landau energy functional,

$$
H(u)(t)=\int_{\mathbb{R}^{d}} \frac{1}{2}|\nabla u(r, t)|^{2}+F(u(r, t)) \mathrm{d} r .
$$

Note that, at low temperature, $\beta^{-1} \ll 1$ and with the choice of $F(q)=\frac{\left(q^{2}-1\right)^{2}}{4},(2.9)$ is the well known Allen-Cahn [1] equation,

$$
u_{t}-\Delta u+u\left(u^{2}-1\right)=0, \quad(r, t) \in \mathbb{R}^{d} \times\{t \geqslant 0\} .
$$

In contrast to the preceding microscopic picture, phenomenological models are reduced descriptions of phase transitions in terms of macroscopic or observable variables such as surface tension and interface mobility. One presupposes that the interface exists as a coherent structure and the order parameter $u$ solves an evolutionary partial differential equation. Sharp-interface or macroscopic models in isothermal systems, which are derived using rigorous continuum mechanics 
arguments [19], represent the interface as a $(d-1)$-dimensional hypersurface $\Gamma_{t}$ in $\mathbb{R}^{d}$ that evolves in time with its local normal velocity

$$
V=v(r, t, n, \nabla n), \quad(r, t) \in \Gamma_{t} \times\{t \geqslant 0\},
$$

where $n$ is the outward unit normal vector to $\Gamma_{t}$, and its curvature tensor $\nabla n$. The function $v$ is specified by a set of constitutive relations. An important isotropic example is the motion by mean curvature

$$
V=-\mu \sigma \operatorname{tr} \nabla n=-\mu \sigma \kappa
$$

with interfacial mobility $\mu$, excess free energy per unit area (surface tension) $\sigma$, and mean curvature $\kappa$.

In general, a hypersurface $\Gamma_{t}$ evolving under law (2.12) may develop geometric singularities, change topology and exhibit various pathologies within a finite time even if the initial interface $\Gamma_{0}$ is smooth. Much work has been done to interpret (2.12) beyond singularities when the classical notion of curvature becomes undefined. First, Brakke [4] provided a weak formulation of (2.13) past singularities by expressing the evolving hypersurface as a varifold. The more general level set method was introduced by Osher and Sethian [30] and was rigorously developed in the context of viscosity solutions by Evans and Spruck [15] and Chen et al. [10]. In this method, the solution $\Gamma_{t}$ of (2.12) is represented as the zero level set of an auxiliary function $\varphi$, i.e. $\Gamma_{t}=\left\{r \in \mathbb{R}^{d}: \varphi(r, t)=\right.$ $0\}$, for all $t \geqslant 0$ past possible singularities, and $\varphi$ solves the geometric partial differential equation

$$
\begin{cases}\varphi_{t}+\mathcal{F}\left(r, t, \nabla \varphi, \nabla^{2} \varphi\right)=0, & (r, t) \in \mathbb{R}^{d} \times\{t \geqslant 0\}, \\ \varphi(x, 0)=\varphi_{0}(r), & r \in \mathbb{R}^{d},\end{cases}
$$

where

$$
\mathcal{F}(r, t, p, X)=-|p| v\left(r, t, \frac{p}{|p|},\left(I-\frac{p \otimes p}{|p|^{2}}\right) X\right)
$$

in the viscosity sense [11]. Here, $p \otimes q$ denotes a $(d \times d)$ matrix with entries $p_{i} q_{j}, p, q \in \mathbb{R}^{d}, I$ is the $(d \times d)$ identity matrix and $X$ is a $(d \times d)$ matrix. The geometric equation corresponding to the mean curvature law (2.13) is

$$
\varphi_{t}-\mu \sigma \operatorname{tr}\left(I-\frac{\nabla \varphi \otimes \nabla \varphi}{|\nabla \varphi|^{2}}\right) \nabla^{2} \varphi=0 .
$$

Under a suitable space-time rescaling, Evans et al. [16] rigorously showed that the Allen-Cahn equation (2.11) yields a front $\Gamma_{t}$ moving under (2.13) or equivalently (2.15) globally in time past singularities. Further results on derivations of geometric laws such as (2.12) from reaction-diffusion equations in the context of viscosity solutions were obtained, for instance, in [8] and [7] (see also references therein). Ilmanen [22] proved that the solution of the Allen-Cahn equation converges to Brakke's mean curvature flow. In [24] and [25], it was shown that the asymptotic behaviour of local mean field equation (2.5) as well as its underlying interacting particle system yield a front moving with normal velocity proportional to a (possibly anisotropic) function of its curvature in the viscosity sense. Additionally, the explicit dependence of macroscopic quantities such as mobility and surface tension on the microscopics was identified via Kubo-Green-type formulae. Similar results were 
obtained in [26] for mesoscopic local mean field equations modelling catalytic surface processes. For an interacting particle system with both Glauber and Kawasaki (spin exchange) dynamics, Katsoulakis and Souganidis [23] proved the existence of suitable space-time scalings such that, in the limit, the sites of the spin system separate into common phase clusters whose boundaries move towards equilibrium under the mean curvature law (2.13), in the Brakke sense.

The foregoing results provide some rigorous justification to a general picture concerning both microscopic and macroscopic approaches to modelling phase transitions: in a large enough sample, individual particles which evolve and interact randomly tend to organize themselves in a coherent deterministic pattern described by a macroscopic equation, say (2.14) at some large space-time scale. Very crudely, this averaging effect is a dynamic version of the law of large numbers. Motivated by this observation, one would hope that, for finer scales of the microscopic models, the local random fluctuations are preserved in the macroscopic equation, similarly to a central limit theorem.

A typical situation which is triggered by random fluctuations but cannot be described by the deterministic macroscopic equation (2.14) or the mesoscopic equations (2.5) and (2.11) is spinodal decomposition. Consider, for example, the Allen-Cahn equation (2.11) with initial datum $u_{0}^{\epsilon}(r) \equiv 0$, $r \in \mathbb{R}^{d}$. Zero is an unstable equilibrium for the vector field of $F^{\prime}(u)$, so the system remains in this state for all times. Suppose a small perturbation $\xi(r, t)$ was added to the equation. Almost surely, $\xi(r, t)$ will perturb the system off of its current unstable zero equilibrium and distinct observable bulk regions of \pm 1 phase clusters will be seen to form for $t>0$. When $\xi$ is random as in (2.9), numerical experiments using the same initial datum may lead to different geometric realizations of these clusters. As proven rigorously in [13], the same phenomenon is observed in the Ising spin model starting at an initial state where the spin average is zero, as for instance when sites alternate spins like a chessboard.

In this paper, we investigate another example where random fluctuations may include useful information. For example, when the initial interface $\Gamma_{0}$ is the union of the coordinate axes in $\mathbb{R}^{2}$, classical solutions to the mean curvature flow (2.13) do not exist. The Brakke solution exists but is not unique, thus giving rise to several evolutions [4]. On the other hand, the zero-level set of the viscosity solution $\Gamma_{t}$ develops an interior, i.e. fattens for $t>0$, containing all possible Brakke solutions, and thereby provides even less information [15]. Angenent et al. [2] have computed a self-similar solution of (2.15) in $\mathbb{R}^{3}$ whose initial zero level set is a smooth surface that fattens in finite time and whose Brakke flow is not unique. See also [3] for other such examples. When an external time-dependent driving term is present, Belletini and Paolini [6] constructed examples of a Lipschitz function $\varphi_{0}: \mathbb{R}^{2} \rightarrow \mathbb{R}$ whose level curves evolve in the viscosity sense according to the law

$$
V=-\mu \sigma \kappa+g(t), \quad(r, t) \in \mathbb{R}^{d} \times\{t \geqslant 0\},
$$

and which develop a full two-dimensional interior in finite time.

In view of the convergence of the Glauber-Kawasaki dynamics to Brakke flows [23], as well as plausible conjectures for other Ising systems along these lines, one hopes that microscopic random models such as the SGL and Ising systems may provide an additional random selection mechanism among all possible weak Brakke solutions, discarding unstable ones, at the points of instability such as self-intersections on the evolving surface. We numerically investigate this conjecture in Sections 5 and 6. First, we will study the manifestation of microscopic fluctuations to macroscopic geometric flows in the formal limit of a suitably rescaled SGL equation (2.9). In addition, we derive a level set formulation for the resulting stochastic curvature flow. 


\section{Formal asymptotics of the SGL model}

In this section, we focus on the asymptotic (long time) behaviour of the continuum version of the SGL equation (2.9):

$$
u_{t}-\Delta u+F_{u}(u)+\sqrt{\frac{2}{\beta}} J(r, t)=0, \quad(r, t) \in \mathbb{R}^{d} \times\{t \geqslant 0\} .
$$

Recall that $F(\cdot)$ is a symmetric double-well potential with global minima $h_{ \pm}$and a local maximum $h_{0}$, and $J$ is a white noise in $\mathbb{R}^{d} \times\{t \geqslant 0\}$. With the scaling $u^{\epsilon}(r, t) \doteq u\left(\epsilon^{-1} r, \epsilon^{-2} t\right)$ and using the scaling property of the Brownian sheet

$$
J\left(\alpha r_{1}, \alpha r_{2}, \ldots, \alpha r_{d}, \gamma t\right)=\alpha^{-\frac{d}{2}} \gamma^{-\frac{1}{2}} J\left(r_{1}, r_{2}, \ldots, r_{d}, t\right),
$$

for scalars $\alpha, \gamma \geqslant 0$ in the distribution sense, (2.9) becomes

$$
u_{t}^{\epsilon}-\Delta u^{\epsilon}+\epsilon^{-2} F_{u^{\epsilon}}\left(u^{\epsilon}\right)+\epsilon^{\frac{d-2}{2}} \sqrt{\frac{2}{\beta}} J(r, t)=0 .
$$

In our analysis, we consider the following rescaling of (2.9):

$$
u_{t}-\Delta u+\epsilon^{-2} F^{\prime}(u)+\epsilon^{-\frac{1}{2}} \sqrt{\frac{2}{\beta}} J\left(r_{1}, r_{2}, \ldots, r_{d}, t\right)=0 .
$$

The reason for this choice of scales will become clear later in the section. The superscript $\epsilon$ is eliminated for clarity. Following the work of Rubinstein et al. [31], we suppose that the order parameter $u$ which solves (3.3) has a formal asymptotic expansion of the form

$$
u(r, t, \epsilon)=p(z, r, \tau, t, \eta)+\epsilon q(z, r, \tau, t, \eta)+\mathrm{O}\left(\epsilon^{2}\right),
$$

where $z=\frac{\phi(r, t, \eta)}{\epsilon}, \tau=\epsilon^{-2} t$ and $\eta=\epsilon^{2} t$. Let $\Gamma_{t} \doteq\left\{r \in \mathbb{R}^{d}: \phi(r, t)=0\right\}$ describe an evolving surface of codimension one in $\mathbb{R}^{d}$. The goal is to derive an evolutionary equation for $\phi$. Since $u$ is stochastic, all derivatives in $(x, t)$ are to be interpreted in the Stratonovich sense. The reason for doing this is that the Stratonovich formula for stochastic differentiation is formulaically identical to the classical chain rule. We refer to Gardiner [18] for a discussion of stochastic integrals in the Ito and Stratonovich sense and their relations, and Walsh [34] for a discussion of the white noise.

We substitute expansion (3.4) into (3.3). Then, matching coefficients of order $\epsilon^{-2}$ leads to

$$
p_{\tau}-p_{z z}|\nabla \phi|^{2}+F^{\prime}(p)=0 .
$$

We assume that as $\tau \uparrow \infty, p$ becomes a travelling wave of one variable, $P(\zeta)=P(z-c \tau)$ where $c$ denotes the wave speed and $P$ connects the two stable minima of $F$, i.e. $P( \pm \infty)=h_{ \pm}$. Equation (3.5) becomes

$$
-c \dot{P}-|\nabla \phi|^{2} \ddot{P}+F^{\prime}(P)=0 .
$$

It is easy to see that $c=[F]\left(\int_{-\infty}^{\infty} \dot{P}^{2} \mathrm{~d} \zeta\right)^{-1}$, where $[F] \doteq F\left(h_{+}\right)-F\left(h_{-}\right)$; and since the wells of $F$ are equally deep, $c=0$. A useful result which will be used in upcoming calculations, see (3.8), is 
the $\zeta$-derivative of (3.5), i.e. $|\nabla \phi|^{2} \dddot{P}-\dot{P} F^{\prime \prime}(P)=0$. Matching the coefficients of order $\epsilon^{-1}$ leads to

$$
p_{z} \phi_{t}-2 \nabla p_{z} \cdot \nabla \phi-p_{z} \Delta \phi+\sqrt{\frac{2}{\beta}} \epsilon^{\frac{1}{2}} J=-q_{\tau}+q_{z z}|\nabla \phi|^{2}-q F^{\prime \prime}(p) .
$$

Note that the noise term $J$ enters (3.6) because an integration of $J$ against $\dot{P}$ at the end of these calculations will absorb the $\epsilon^{\frac{1}{2}}$ factor: see comments immediately preceding (3.10). Again, we assume that as $\tau \uparrow \infty, q$ is a function of one variable, $q=Q(\zeta)$, to obtain

$$
\left(\phi_{t}-\Delta \phi\right) \dot{P}-2 \nabla \dot{P} \cdot \nabla \phi+\sqrt{\frac{2}{\beta}} \epsilon^{\frac{1}{2}} J=\ddot{Q}|\nabla \phi|^{2}-Q F^{\prime \prime}(P) .
$$

The right-hand side of equality (3.7) is the linearized travelling wave operator around $P$. By Fredholm's alternative, (3.7) is formally solvable if and only if its left-hand side is orthogonal to any kernel element of the dual of the linearized operator. This kernel is the linear span of $\dot{P}$. Multiplying (3.7) by $\dot{P}$, then integrating by parts over $\zeta \in \mathbb{R}$, and using the $\zeta$-derivative of (3.5) leads to

$$
\begin{aligned}
\left(\phi_{t}-\Delta \phi\right) \int_{-\infty}^{\infty} \dot{P}^{2} \mathrm{~d} \zeta & -\nabla\left(\int_{-\infty}^{\infty} \dot{P}^{2} \mathrm{~d} \zeta\right) \cdot \nabla \phi \\
& +\sqrt{\frac{2}{\beta}} \epsilon^{\frac{1}{2}} \int_{-\infty}^{\infty} J \dot{P} \mathrm{~d} \zeta=0
\end{aligned}
$$

Define a change of variables $s \doteq \frac{\zeta}{|\nabla \phi|}$ and set $\mathbb{P}(s) \doteq P(\zeta)$. Note that $\int_{-\infty}^{\infty} \dot{P}^{2}(\zeta) \mathrm{d} \zeta|\nabla \phi|=$ $\int_{-\infty}^{\infty} \mathbb{P}_{s}^{2}(s) \mathrm{d} s$ is independent of $r$, and $\mathbb{P}$ is the standing wave solving $\mathbb{P}_{s s}-F^{\prime}(\mathbb{P})=0$. Thus, (3.8) becomes

$$
\begin{aligned}
\phi_{t} & -\Delta \phi+\frac{\nabla \phi \cdot \nabla|\nabla \phi|}{|\nabla \phi|} \\
& +|\nabla \phi| \sqrt{\frac{2}{\beta}}\left(\int_{-\infty}^{\infty} \mathbb{P}_{s}^{2}(s) \mathrm{d} s\right)^{-1} \epsilon^{\frac{1}{2}} \int_{-\infty}^{\infty} J(x, t) \mathbb{P}_{s}(s) \mathrm{d} s=0 .
\end{aligned}
$$

Following Spohn [32], the interface mobility $\mu$ and its surface tension $\sigma$ are defined as

$$
\mu \doteq\left(\int_{-\infty}^{\infty} \mathbb{P}_{s}^{2}(s) \mathrm{d} s\right)^{-1}, \quad \sigma \doteq \int_{-\infty}^{\infty} \mathbb{P}_{s}^{2}(s) \mathrm{d} s
$$

The final step in deriving an equation for $\phi$ is to unravel the random integral in (3.9). It is clear that close to the interface $\Gamma_{t}, \epsilon s=\frac{\phi}{|\nabla \phi|}$ has order $\mathrm{O}(1)$ and behaves like a signed distance function to $\Gamma_{t}$. By this observation, the integral in (3.9) denotes an integration of the travelling wave $\mathbb{P}$ in the direction perpendicular to $\Gamma_{t}$, after which the resulting random term will be solely supported on $\Gamma_{t}$. Without loss of generality, we may assume that the $r_{1}$-coordinate of $r$ is orthogonal to the tangent 
space of $\Gamma_{t}$ at $r$. Restricting $(r, t)$ to $\Gamma_{t}$ and letting $r_{1}=\epsilon s$ for $\epsilon \ll 1$ produces

$$
\begin{aligned}
\int_{-\infty}^{\infty} J\left(r_{1}, r_{2}, \ldots, r_{d}, t\right) \mathbb{P}_{s}(s) \mathrm{d} s & =\int_{-\infty}^{\infty} J\left(\epsilon s, r_{2}, \ldots, r_{d}, t\right) \mathbb{P}_{s}(s) \mathrm{d} s \\
& =\epsilon^{-\frac{1}{2}} \int_{-\infty}^{\infty} J\left(s, r_{2}, \ldots, r_{d}, t\right) \mathbb{P}_{s}(s) \mathrm{d} s .
\end{aligned}
$$

Therefore, (3.9) becomes

$$
\begin{gathered}
\phi_{t}-\mu \sigma \operatorname{tr}\left(\left(I-\frac{\nabla \phi \otimes \nabla \phi}{|\nabla \phi|^{2}}\right) \nabla^{2} \phi\right) \\
+|\nabla \phi| \sqrt{\frac{2}{\beta}} \mu \int_{-\infty}^{\infty} J\left(s, r_{2}, \ldots, r_{d}, t\right) \mathbb{P}_{s}(s) \mathrm{d} s=0, \\
(r, t) \in \Gamma_{t} \times\{t \geqslant 0\} .
\end{gathered}
$$

Note that the mean curvature $\kappa=\frac{1}{|\nabla \phi|} \operatorname{tr}\left(\left(I-\frac{\nabla \phi \otimes \nabla \phi}{|\nabla \phi|^{2}}\right) \nabla^{2} \phi\right)$. Next, we define the noise:

$$
\begin{gathered}
\mathcal{J}(\rho, t) \doteq \sqrt{\mu} \int_{-\infty}^{\infty} J\left(s, \rho_{2}, \ldots, \rho_{d}, t\right) \mathbb{P}_{s}(s) \mathrm{d} s \\
\rho=\left(\rho_{2}, \ldots, \rho_{d}\right) \in \mathbb{R}^{d-1} .
\end{gathered}
$$

The covariance of (3.11) is

$$
\begin{aligned}
\left\langle\mathcal{J}(\rho, t), \mathcal{J}\left(\rho^{\prime}, t^{\prime}\right)\right\rangle= & \mu\left\langle\left(\int_{-\infty}^{\infty} J\left(s, \rho_{2}, \ldots, \rho_{d}, t\right) \mathbb{P}_{s}(s) \mathrm{d} s\right),\right. \\
& \left.\left(\int_{-\infty}^{\infty} J\left(s^{\prime}, \rho_{2}^{\prime}, \ldots, \rho_{d}^{\prime}, t^{\prime}\right) \mathbb{P}_{s^{\prime}}\left(s^{\prime}\right) \mathrm{d} s^{\prime}\right)\right\rangle \\
= & \mu \int_{-\infty}^{\infty} \mathbb{P}_{s}^{2}(s) \mathrm{d} s \delta\left(\rho_{2}-\rho_{2}^{\prime}\right) \ldots \delta\left(\rho_{d}-\rho_{d}^{\prime}\right) \delta\left(t-t^{\prime}\right) \\
= & \delta\left(\rho_{2}-\rho_{2}^{\prime}\right) \ldots \delta\left(\rho_{d}-\rho_{d}^{\prime}\right) \delta\left(t-t^{\prime}\right), \\
& (\rho, t),\left(\rho^{\prime}, t^{\prime}\right) \in \mathbb{R}^{d-1} \times\{t \geqslant 0\},
\end{aligned}
$$

where $\delta(\cdot)$ is the Dirac measure on $\mathbb{R}$. Using (3.11), we may rewrite (3.10) as

$$
\begin{gathered}
\phi_{t}-\mu \sigma \operatorname{tr}\left(\left(I-\frac{\nabla \phi \otimes \nabla \phi}{|\nabla \phi|^{2}}\right) \nabla^{2} \phi\right)+|\nabla \phi| \sqrt{\frac{2 \mu}{\beta}} \mathcal{J}(\varrho, t)=0, \\
(\varrho, t) \in \Gamma_{t} \times\{t \geqslant 0\}, \quad \varrho=\left(r_{2}, \ldots, r_{d}\right) .
\end{gathered}
$$

Note that the stochastic term in (3.13) is not Gaussian, namely its covariance is not deterministic when it is considered on $\Gamma_{t}$ due to the fluctuations of $\Gamma_{t}$. The stochastic partial differential equation (3.13) implies that $\Gamma_{t}$ evolves with the local outward normal velocity,

$$
V=-\mu \sigma \kappa+\sqrt{\frac{2 \mu}{\beta}} \mathcal{J}(\varrho, t), \quad(\varrho, t) \in \Gamma_{t} \times\{t \geqslant 0\} .
$$


Our initial choice of scaling in (3.3) allows us to derive this macroscopic limit (3.14) with the random effect present at the same $\epsilon$ order as the deterministic curvature effect. The scaling (3.2) will yield the stochastic term as a higher-ordered perturbation of the motion by mean curvature (2.13). We refer to (3.14) as stochastic mean curvature flow.

The law (3.14) was first formally derived by Kawasaki and Ohta [27] and Bausch et al. [5] from the SGL model in the case when the propagating interface is a graph over $\mathbb{R}^{d-1}$. If we suppose that the propagating surface in $\mathbb{R}^{d}$ is given as a graph over $\mathbb{R}^{d-1},\left(r^{\prime}, f\left(r^{\prime}, t\right)\right), r^{\prime}=\left(r_{1}, \ldots, r_{d-1}\right)$, then the auxiliary function $\phi$ for its equivalent level set formulation is set to $r_{d}-f\left(r^{\prime}, t\right), \quad r=$ $\left(r_{1}, \ldots, r_{d}\right)$, and $\phi$ solves (3.13) for $(\varrho, t) \in \Gamma_{t} \times\{t \geqslant 0\}$. Since $\mathrm{d} \varrho=\sqrt{1+|\nabla f|^{2}} \mathrm{~d} r^{\prime}$, property (3.1) gives

$$
\begin{aligned}
\mathcal{J}(\varrho, t) \mathrm{d} \varrho \mathrm{d} t=W(\mathrm{~d} \varrho, \mathrm{d} t) & =\left(1+|\nabla f|^{2}\right)^{\frac{1}{4}} W\left(\mathrm{~d} r^{\prime}, \mathrm{d} t\right) \\
& =\left(1+|\nabla f|^{2}\right)^{\frac{1}{4}} J\left(r^{\prime}, t\right) \mathrm{d} r^{\prime} \mathrm{d} t
\end{aligned}
$$

where $J\left(r^{\prime}, t\right)$ is now a white noise on $\mathbb{R}^{d-1} \times\{t \geqslant 0\}$. In this setting, (3.13) becomes

$$
\begin{aligned}
f_{t}-\mu \sigma \sqrt{1+|\nabla f|^{2}} & \operatorname{div}\left(\frac{\nabla f}{\sqrt{1+|\nabla f|^{2}}}\right) \\
& +\sqrt{\frac{2 \mu}{\beta}}\left(1+|\nabla f|^{2}\right)^{\frac{1}{4}} J\left(r^{\prime}, t\right)=0,
\end{aligned}
$$

which is the graph equivalent characterization in [27] and [5]. Note that by the fluctuationdissipation theorem $[5,18]$, the dynamics of (3.15) leave the underlying Gibbs distribution function

$$
Z^{-1} \exp \left(-\beta \int_{-\infty}^{\infty} \sigma \sqrt{1+|\nabla f|^{2}} \mathrm{~d} r^{\prime}\right)
$$

invariant, similarly to the microscopic models in Section 2. We refer to Yip [35] for the existence of interface evolutions driven by coloured noise using a flat-flow formulation. Lions and Souganidis [29] have provided a viscosity solution framework for general fully nonlinear stochastic partial differential equations driven by a coloured noise. Furthermore, the convergence of the AllenCahn model to stochastic motion by mean curvature for white noise in time only was rigorously established by Funaki [17] when $d=2$, and by Lions and Souganidis [29] for any $d$.

\section{A level set formulation for (3.14)}

We now proceed to define a suitable level set formulation for the stochastic mean curvature flow (3.14). The level set formulation for the normal velocity law

$$
V=-\mu \sigma \kappa+c(r, t), \quad(r, t) \in \Gamma_{t} \times\{t \geqslant 0\},
$$

where $c(r, t)$ is a Lipschitz-continuous function of $(r, t)$ is well known [8]. Its corresponding front $\Gamma_{t}$ is the zero level set of a function $\varphi$ which solves

$$
\begin{gathered}
\varphi_{t}-\mu \sigma \operatorname{tr}\left(\left(I-\frac{\nabla \varphi \otimes \nabla \varphi}{|\nabla \varphi|^{2}}\right) \nabla^{2} \varphi\right)+|\nabla \varphi| c(r, t)=0, \\
(r, t) \in \mathbb{R}^{d} \times\{t \geqslant 0\}
\end{gathered}
$$


in the viscosity sense.

Observe that by merely setting $c(r, t)=J(r, t)$, where $J$ is a white noise over $\mathbb{R}^{d} \times\{t \geqslant$ $0\}$, see (2.10), the restriction of $J$ onto any $(d-1)$-dimensional hypersurface (and consequently onto $\Gamma_{t}$ ) is random with an infinite covariance function and does not satisfy (3.12). To see this, consider a projection of $J(r, t)$ onto any hyperplane $\left\{r_{1}=\lambda\right\}$ where $\lambda$ is a constant, noting that $\left\langle J(r, t), J\left(r^{\prime}, t^{\prime}\right)\right\rangle=\delta\left(r_{1}-r_{1}^{\prime}\right) \ldots \delta\left(r_{d}-r_{d}^{\prime}\right) \delta\left(t-t^{\prime}\right)$, for $(r, t),\left(r^{\prime}, t^{\prime}\right) \in \mathbb{R}^{d} \times\{t \geqslant 0\}$. In view of this difficulty, we will define the coloured noise $\mathcal{J}^{\gamma}=\mathcal{J}^{\gamma}(r, t),(r, t) \in \mathbb{R}^{d} \times\{t \geqslant 0\}$, with covariance

$$
\begin{aligned}
& \left\langle\mathcal{J}^{\gamma}(r, t), \mathcal{J}^{\gamma}\left(r^{\prime}, t^{\prime}\right)\right\rangle=\gamma^{-d} \Phi\left(\frac{r_{1}-r_{1}^{\prime}}{\gamma}\right) \cdots \Phi\left(\frac{r_{d}-r_{d}^{\prime}}{\gamma}\right) \Phi\left(\frac{t-t^{\prime}}{\gamma}\right), \\
& (r, t),\left(r^{\prime}, t^{\prime}\right) \in \mathbb{R}^{d} \times\{t \geqslant 0\}, \quad \Phi(s) \doteq \sqrt{\pi} \exp \left(-s^{2}\right), \quad s \in \mathbb{R} .
\end{aligned}
$$

Note that the standard regularization of a $d$-dimensional white noise $J(r, t)$ would have covariance $\gamma^{-(d+1)} \Phi\left(\frac{r_{1}-r_{1}^{\prime}}{\gamma}\right) \ldots \Phi\left(\frac{r_{d}-r_{d}^{\prime}}{\gamma}\right) \Phi\left(\frac{t-t^{\prime}}{\gamma}\right)$. Due to the $\gamma$-scaling choice in (4.2) and the isotropy of $\Phi$, the projection of $\mathcal{J}^{\gamma}$ onto a $(d-1)$-dimensional hypersurface gives rise to a $(d-1)$-dimensional white noise on the hypersurface as $\gamma \downarrow 0$. A trivial example is the projection of $\mathcal{J}^{\gamma}$ onto the hyperplane $\left\{r_{1}=\lambda\right\}$ which yields a white noise in $\mathbb{R}^{d-1}$ as $\gamma \downarrow 0$.

Using $\mathcal{J}^{\gamma}$, we will approximate (3.14) with the level set formulation

$$
\begin{gathered}
\phi_{t}^{\gamma}-\mu \sigma \operatorname{tr}\left(\left(I-\frac{\nabla \phi^{\gamma} \otimes \nabla \phi^{\gamma}}{\left|\nabla \phi^{\gamma}\right|^{2}}\right) \nabla^{2} \phi^{\gamma}\right)+\left|\nabla \phi^{\gamma}\right| \sqrt{\frac{2 \mu}{\beta}} \mathcal{J}^{\gamma}(r, t)=0, \\
(r, t) \in \mathbb{R}^{d} \times\{t \geqslant 0\} .
\end{gathered}
$$

The approximating random interface is defined as $\Gamma_{t}^{\gamma} \doteq\left\{r \in \mathbb{R}^{d}: \phi^{\gamma}(r, t)=0\right\}$. By replacing $\gamma^{-1} \Phi\left(\frac{t-t^{\prime}}{\gamma}\right)$ with $\delta\left(t-t^{\prime}\right)$ in (4.2), we can define (4.2) rigorously via a Stratonovich integral and consider suitable weak solutions for (4.3) in the viscosity sense [29].

We briefly comment upon the numerical implementation of (4.3). The deterministic parts of (4.3), namely (2.15), are handled by methods detailed in [30] (see also [33]). The coloured noise $\mathcal{J}^{\gamma}(r, t)$ can be approximated by

$$
\mathcal{J}^{\gamma}(r, t) \approx \Delta x^{\frac{1}{2}}\left(\Delta t \Delta x^{-d}\right)^{\frac{1}{2}} \eta_{t}(x), \quad \Delta x=\gamma, \quad r \approx x,
$$

where $\eta_{t}(x)$ is a normally distributed random variable with mean zero and variance one; $\eta_{t}(x)$ and $\eta_{t}(y)$ are independent when $x \neq y$ where $x$ and $y$ are points on the computational grid, and $\Delta x$ is the uniform space grid size. This approximation will be further clarified in the context of the SGL system in Section 5.

\section{Numerical experiments using the SGL scheme}

Here, we numerically investigate the effect of random fluctuations to motion by mean curvature for planar curves. First, we present the approximating scheme to the SGL equation (3.2) which will be used in the experiments. Let the potential $F(q)=\frac{\left(q^{2}-1\right)^{2}}{4}$. Recall that (3.2), set on a bounded 
domain $\mathcal{O} \subset \mathbb{R}^{2}$, reads

$$
\left\{\begin{array}{lr}
u_{t}(r, t)-\Delta u(r, t)+\epsilon^{-2} F^{\prime}(u(r, t))+\sqrt{\frac{2}{\beta}} \epsilon^{\frac{d-2}{2}} J(r, t)=0, \\
u(r, 0)=u_{0}(r), & (r, t) \in \mathcal{O} \times\{t>0\} .
\end{array}\right.
$$

From the asymptotic analysis of Section 3 (see comments immediately following (3.14)), when $\epsilon \ll 1$, the zero level set of $u$ moves approximately according to the stochastic mean curvature flow

$$
V=-\mu \sigma \kappa+\sqrt{\frac{2 \mu}{\beta}} \epsilon^{\frac{d-1}{2}} \mathcal{J} .
$$

Consider a uniform space discretization of size $\Delta x$ for (5.1):

$$
\begin{aligned}
\frac{\mathrm{d}}{\mathrm{d} t} u(x, t)-\Delta_{\Delta x} u(x, t) & +\epsilon^{-2} F^{\prime}(u(x, t)) \\
& +\sqrt{\frac{2}{\beta}} \epsilon^{\frac{d-2}{2}}\left(\frac{1}{\Delta x}\right)^{\frac{d}{2}} \frac{\mathrm{d}}{\mathrm{d} t} w(x ; t)=0, \\
(x, t) & \in \mathcal{O}_{\Delta x} \times\{t>0\},
\end{aligned}
$$

where $w(x ; t)$ are independent Brownian motions for each $x \in \mathcal{O}_{\Delta x}$ and

$$
\begin{aligned}
\mathcal{O}_{\Delta x} & \doteq\left\{\left(i_{1} \Delta x, \ldots, i_{d} \Delta x\right) \in \mathcal{O}: i_{1}, \ldots, i_{d} \in\{1, \ldots, M-1\}\right\}, \\
\overline{\mathcal{O}}_{\Delta x} & \doteq\left\{\left(i_{1} \Delta x, \ldots, i_{d} \Delta x\right) \in \mathcal{O}: i_{1}, \ldots, i_{d} \in\{0,1, \ldots, M\}\right\}, \\
\partial \mathcal{O}_{\Delta x} & \doteq \overline{\mathcal{O}}_{\Delta x} \backslash \mathcal{O}_{\Delta x} \text { (i.e. the boundary points), } \\
\Delta_{\Delta x} u(x, t) & \doteq \frac{\sum_{j=1}^{d} u\left(x+\Delta x e_{j}, t\right)+u\left(x-\Delta x e_{j}, t\right)-2 u(x, t)}{\Delta x^{2}}
\end{aligned}
$$

where $\left\{e_{j}\right\}_{j=1}^{d}$ is the standard basis in $\mathbb{R}^{d}$. Note that (5.3) is a system of stochastic differential equations for $u(\cdot, t)$ indexed by $x \in \mathcal{O}_{\Delta x}$. The noise coefficient $(\Delta x)^{-\frac{d}{2}}$ arises from the formal variance of $\frac{\partial}{\partial x_{j}} W(x, t), x \in \mathcal{O}_{\Delta x}$, being approximated by $\operatorname{var}\left(\frac{W\left(x+\Delta x e_{j}, t\right)-W(x, t)}{\Delta x}\right)=(\Delta x)^{-1}$, where $W$ is a Brownian sheet as defined in (2.10). Next, we discretize (5.3) in the time variable. Let $T>0$ be some finite time, $\Delta t$ be the uniform time step size and define

$$
\begin{aligned}
\Sigma_{\Delta t} & \doteq\{k \Delta t \in[0, T]: k \in\{0,1, \ldots, N\}\}, \\
U_{x}^{k} & \doteq u(x, k \Delta t), \quad(x, k \Delta t) \in \mathcal{O}_{\Delta x} \times \Sigma_{\Delta t} .
\end{aligned}
$$

The Brownian motion $w(x ; t)$ is approximated from the left endpoint of each discrete time interval. At every $x, \delta_{k \Delta t} w(x) \doteq w(x ;(k+1) \Delta t)-w(x ; k \Delta t)$ are independent, normally distributed random variables of mean zero and variance $\Delta t$. Following Kloeden and Platen [28], a time-explicit Euler scheme for stochastic differential equations is used:

$$
\begin{gathered}
\left(U_{x}^{k+1}-U_{x}^{k}\right)-\Delta_{\Delta x} U_{x}^{k} \Delta t+\epsilon^{-2} F^{\prime}\left(U_{x}^{k}\right) \Delta t \\
+\sqrt{\frac{2}{\beta}} \epsilon^{\frac{d-2}{2}}\left(\frac{1}{\Delta x}\right)^{\frac{d}{2}} \delta_{k \Delta t} w(x)=0 \\
(x, k \Delta t) \in \mathcal{O}_{\Delta x} \times \Sigma_{\Delta t} .
\end{gathered}
$$


Boundary conditions will be specified later. In practice, (5.4) is implemented as

$$
\begin{aligned}
U_{x}^{k+1}=(1-2 d \mu) U_{x}^{k} & +\mu \sum_{j=1}^{d}\left(U_{x-\Delta x e_{j}}^{k}+U_{x+\Delta x e_{j}}^{k}\right)-\frac{\Delta t}{\epsilon^{2}} F^{\prime}\left(U_{x}^{k}\right) \\
& -\sqrt{\frac{2}{\beta}}\left(\frac{\epsilon^{d-2} \Delta t}{\Delta x^{d}}\right)^{\frac{1}{2}} \eta_{k} \Delta t(x),
\end{aligned}
$$

where $\mu \doteq \frac{\Delta t}{\Delta x^{2}}$ and $\eta_{k} \Delta t(x)$ is a normally distributed random variable of mean zero and variance one with $\eta_{k \Delta t}(x)$ and $\eta_{k \Delta t}(y)$ being independent whenever $x \neq y$. Independence in each $(x, k)$ is guaranteed by re-seeding the pseudo-random number generator.

We now define $\left\|U^{k}\right\|_{\ell^{2}}^{2} \doteq \sum_{x \in \mathcal{O}_{\Delta x}}\left(U_{x}^{k}\right)^{2}$, i.e. the $\ell^{2}$-norm of the approximating order parameter $U^{k}$. Suppose that $U_{x}^{k}$ and $\bar{U}_{x}^{k}$ solve (5.5) with the same stochastic term corresponding to initial data $U_{x}^{0}$ and $\bar{U}_{x}^{0}$. The $\ell^{2}$-stability in the strong sense of [28] for the scheme (5.4) is established by the following theorem.

THEOREM 5.1 Suppose that $F^{\prime}$ is globally Lipschitz continuous, and $U=\bar{U}$ on $\partial \mathcal{O}_{\Delta x} \times \Sigma_{\Delta t}$. Then, there exists a constant $C=C(F, \epsilon)>0$ such that $\sup _{0 \leqslant k \leqslant N} \mathrm{E}\left\|U^{k}-\bar{U}^{k}\right\|_{\ell^{2}} \leqslant C \mathrm{E} \| U^{0}-$ $\bar{U}^{0} \|_{\ell^{2}}$.

Proof. See the Appendix.

Note that the Lipschitz condition on $F^{\prime}$ is not necessary when the solutions $U$ and $\bar{U}$ are bounded. The Lipschitz condition does not cover the typical cubic nonlinearity in the Allen-Cahn model; however, it covers the case when $F$ grows linearly at infinity.

Similarly to simulations done by Elliott [14] and Chen et al. [9] for the deterministic Allen-Cahn equation (2.11), $\Delta x, \Delta t$ and $\mu$ are here picked to satisfy conditions

$$
\left\{\begin{array}{l}
\mu \leqslant\left(2 d+\left(\frac{\Delta x}{\epsilon}\right)^{2} A\right)^{-1}, \\
\Delta x \leqslant \epsilon^{p},
\end{array}\right.
$$

where $A \doteq \sup _{q \in[-\sqrt{2}, \sqrt{2}]}\left|F^{\prime \prime}(q)\right|=5$ and $p$ is any constant greater than one. Condition (5.6) was used in [9] to prove the convergence of the scheme (5.4), without the random component, to mean curvature flow; we note that when (5.6) is violated, the scheme (5.4) becomes unstable.

In our simulations, the computational domain is a rectangular lattice in $\mathbb{R}^{d}, d=2$, and the initial datum $\Gamma_{0}$ is a curve in $\mathcal{O} \subset \mathbb{R}^{2}$. For expositional clarity, suppose that $\Gamma_{0}$ is a simply connected, closed curve. Denote the region inside $\Gamma_{0}$ as $\Omega_{0}^{+}$, and the region outside $\Gamma_{0}$ as $\Omega_{0}^{-}$. Without loss of generality, let $\Omega_{0}^{+}$be the subset of $\mathcal{O}$ which is in phase +1 , and $\Omega_{0}^{-}$be the subset of $\mathcal{O}$ which is in phase -1 . Thus $\Gamma_{0}$ is the antiphase boundary and $\mathcal{O}=\Gamma_{0} \cup \Omega_{0}^{-} \cup \Omega_{0}^{+}$. As in [9], both discontinuous and continuous initial data will be tried. A typical example of a discontinuous datum is

$$
U_{x}^{0}=u_{0}(x)= \begin{cases}+1, & \text { if } x \in \Omega_{0}^{+} \cap \mathcal{O}_{\Delta x} \\ -1, & \text { if } x \in \Omega_{0}^{-} \cap \mathcal{O}_{\Delta x},\end{cases}
$$

while its continuous counterpart might look like

$$
U_{x}^{0}=u_{0}(x)=\tanh \left(\frac{c(x)}{\sqrt{2} \epsilon}\right), \quad x \in \mathcal{O}_{\Delta x},
$$


for a continuous function $c$ that is positive on $\Omega_{0}^{+}$and negative on $\Omega_{0}^{-}$. Note that $\tanh \left(\frac{z}{\sqrt{2}}\right)$ is the standing wave solution to the Allen-Cahn equation (2.11). The thickness of the interface between the \pm 1 phases is related to $\epsilon$, see [14] and references therein. Except for Example 5.8, all initial curves $\Gamma_{0}$ in the upcoming experiments self-intersect.

In each experiment, we track the evolution of the zero level set of $U_{x}^{k}$,

$$
\Gamma_{k \Delta t} \doteq\left\{x \in \mathcal{O}_{\Delta x}: U_{x}^{k}=0, k \in\{0,1,2, \ldots\}\right\} .
$$

In practice, it suffices to track the set $\left\{x \in \overline{\mathcal{O}}_{\Delta x}: U_{x}^{k} \geqslant 0\right\}$ whose boundary is $\Gamma_{k \Delta t}$. With the presence of random fluctuations, $\Gamma_{k} \Delta t$ will almost always look jagged and is not a smooth curve. For small enough temperature $\left(\beta^{-1}\right)$ however, random fluctuations are proportionally small and the bulk regions of phases \pm 1 are visibly distinct. Almost surely, noise will produce different evolutionary realizations with the same initial datum, $U_{x}^{0}$. Specifically, our main interest in these experiments will be to observe both

- the qualitative (topological) behaviour of $\Gamma_{k \Delta t}$ about its self-intersection point, and

- the effect of small perturbations on $\Gamma_{0}$ upon the subsequent evolution of $\Gamma_{k \Delta t}$,

in the presence and absence of the stochastic term in the algorithm (5.5).

For all SGL-scheme experiments, we fix

$$
p=1.16, \quad \mu=0.05, \quad \beta=0.5 \times 10^{9} .
$$

Such a low temperature $\beta^{-1}$ is picked because at higher values the effect of randomness is too pronounced, thus preventing us from confidently distinguishing between phases in $\mathcal{O}_{\Delta x}$. Unless otherwise noted, $\epsilon$ is set to 0.05 . Recall that $\Delta x=\epsilon^{p}$ and $\Delta t=\mu(\Delta x)^{2}$, satisfying conditions (5.6).

A note on the boundary conditions: zero Neumann boundary conditions are applied whenever $\Gamma_{k \Delta t}$ intersects the computational domain boundary $\partial \mathcal{O}_{\Delta x}$ at angle $\frac{\pi}{2}$, or when $\Gamma_{k \Delta t} \cap \partial \mathcal{O}_{\Delta x}=$ $\emptyset$. In Examples 5.2 and 5.4, where $\Gamma_{0}$ meets the top and bottom domain boundary at angle $\frac{\pi}{4}$, a capillary-type boundary condition, $\frac{\partial u}{\partial n}=|\nabla u| \cos \theta$, is used on these respective boundaries, with $n$ being the unit normal to $\partial \mathcal{O}_{\Delta x}$ and $\theta$ being the angle between $\Gamma_{k \Delta t}$ and $\partial \mathcal{O}_{\Delta x}$. This condition preserves a constant angle $\theta$ between $\Gamma_{k \Delta t}$ and $\partial \mathcal{O}_{\Delta x}$ through all iterations.

EXAMPLE $5.1 \Gamma_{0}$ consists of the union of the coordinate axes in $\mathbb{R}^{2}$.

The lattice consists of $100 \times 100$ points centred at $(0,0)$ on the $r_{1} r_{2}$ plane with zero Neumann boundary conditions and the initial datum is $U_{x}^{0}=\tanh \left(\frac{x_{1} x_{2}}{\sqrt{2} \epsilon}\right)$. When the random term in (5.5) is removed, the evolution becomes indefinitely 'suspended', i.e. $\Gamma_{k} \Delta t=\Gamma_{0}$ for all further iterations $k$, see Fig. 1 . When randomness is present, two qualitatively different outcomes were observed corresponding to different realizations:

(A) phase +1 regions join up or equivalently phase -1 regions separate, or

(B) phase +1 regions separate or equivalently phase -1 regions join up.

State suspension is not observed, see Fig. 1. In the random case, we find that these two outcomes have almost equal probability 0.5 of occurring in any one experiment. For instance, in a batch of 50 experiments, 26 yield outcome (A) and 24 (B). 

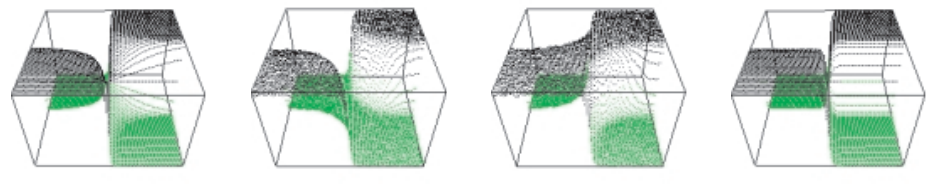

$\mathrm{t}=0.2396$

$\mathrm{t}=0.2396$

$\mathrm{t}=0.2396$
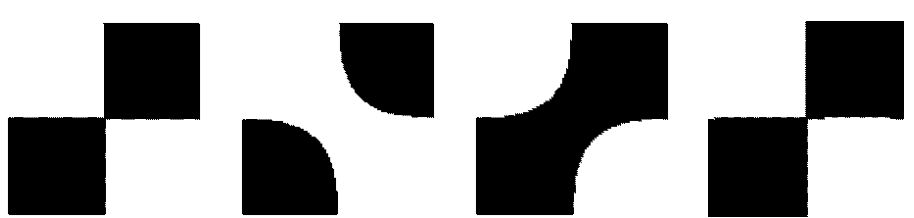

FIG. 1. Example 5.1. Top row, left to right: plot of the order parameter $U_{x}^{k}$ : Initial datum, random evolution (B) (+1 break), random evolution (A) ( +1 join), state suspension in the absence of randomness. Bottom row: black denotes regions where $U_{x}^{k}>0$.

When the initial datum is perturbed at $(0,0)$ by changing $U_{(0,0)}^{0}$ from $U_{(0,0)}^{0}=0.0$ to $U_{(0,0)}^{0}=$ 0.1 , the +1 phase joined when evolved with the deterministic version of (5.5). However, when the random term was present, the above two outcomes (A) and (B) were again observed with about the same probabilities. For instance, in a batch of 50 experiments, 27 resulted in the +1 phases separating, and 23 had the +1 phases joining.

Next we used a discontinuous initial datum. When randomness is absent from the SGL scheme (5.5), the +1 phase separates and when $U^{0}$ is perturbed as above, the subsequent evolution shows the +1 phase joining. As when a continuous initial datum was used, we observe radically different subsequent evolutions with the deterministic scheme when this datum is perturbed. In the presence of randomness, the discontinuous datum produced results that were identical (i.e. the above two outcomes (A) and (B) with approximately equal probability) to the experiments with continuous datum regardless of the perturbation of $U^{0}$. Thus, essentially the same qualitative pictures were observed when a discontinuous datum was used instead of a continuous one.

EXAMPLE $5.2 \Gamma_{0}$ of Example 5.1 is rotated by $\frac{\pi}{4}$.

The lattice consists of $210 \times 200$ points centred at $(0,0)$ on the $r_{1} r_{2}$ plane. The datum is $U_{x}^{0}=$ $\tanh \left(\frac{x_{2}^{2}-x_{1}^{2}}{\sqrt{2} \epsilon}\right)$. Since $\Gamma_{0}$ intersects the top and bottom boundaries at $\frac{\pi}{4}$, a capillary boundary condition with $\theta=\frac{\pi}{4}$ is used there. A zero Neumann condition is applied on the left and right boundaries since $\Gamma_{k \Delta t}$ does not intersect these boundaries for small times.

In the absence of the stochastic component in (5.5), the +1 phase joined. When $U_{(0,0)}^{0}$ is perturbed from 0.0 to -0.1 , the +1 phase separates in the subsequent evolution. With the stochastic component in the scheme, two qualitatively different outcomes were observed as in Example 5.1 with almost equal 0.5 probability, see Fig. 2 . These probabilities appear unaffected by the perturbation of $U^{0}$. Similar qualitative pictures were observed when a discontinuous datum was used instead of a continuous one.

EXAMPLE 5.3 $\Gamma_{0}$ of Example 5.1 is translated in the $r_{1}$ direction.

The lattice consists of $100 \times 100$ points centred at $(0,0)$ on the $r_{1} r_{2}$ plane with zero Neumann boundary conditions and $U_{x}^{0}=\tanh \left(\frac{x_{1}\left(x_{2}+0.2\right)}{\sqrt{2} \epsilon}\right)$. 


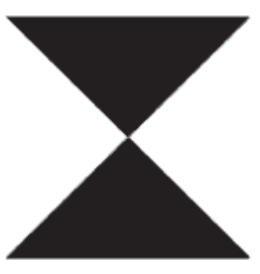

$\mathrm{t}=0$

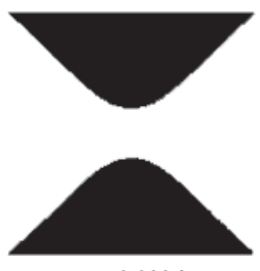

$\mathrm{t}=0.2396$

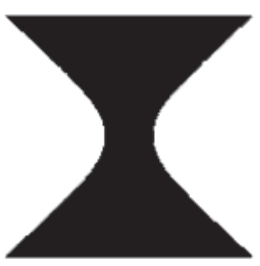

$\mathrm{t}=0.2396$

FIG. 2. Example 5.2. Initial datum and two equiprobable stochastic evolutions. Black denotes regions where $U_{x}^{k}>0$.

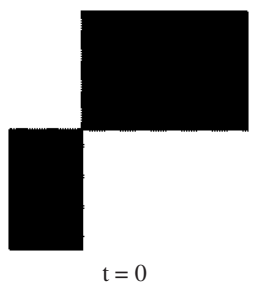

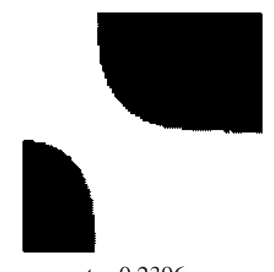

$\mathrm{t}=0.2396$

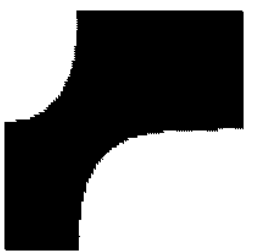

$\mathrm{t}=0.2396$

FIG. 3. Example 5.3. Initial datum and two equi-probable noisy evolutions. Black denotes regions where $U_{x}^{k}>0$.

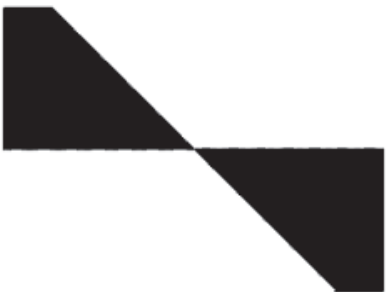

$\mathrm{t}=0$
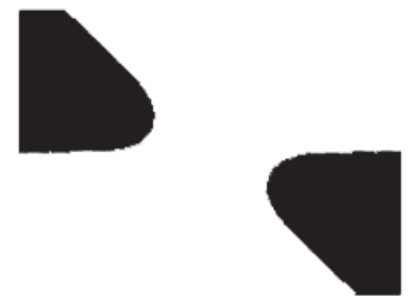

$\mathrm{t}=0.2396$

FIG. 4. Example 5.4. Initial datum and the only possible random evolution. Black denotes regions where $U_{x}^{k}>0$.

In the absence of randomness in (5.5), the +1 phase joins. When $U_{(0,-0.2)}^{0}$ is perturbed from 0.0 to -0.1 , the +1 phase separates. With the presence of randomness, two qualitatively different outcomes were recorded as in Example 5.1, each occurring with almost equal probability 0.5, regardless of the one-point perturbation, see Fig. 3. Similar qualitative pictures were observed when a discontinuous datum was used instead of a continuous one.

EXAMPLE 5.4 $\Gamma_{0}$ consists of two lines intersecting with angle $\frac{\pi}{4}$ at $(0,0)$.

The lattice consists of $200 \times 150$ points centred at $(0,0)$ on the $r_{1} r_{2}$ plane with zero Neumann boundary conditions and $U_{x}^{0}=\tanh \left(\frac{-x_{2}\left(x_{2}+x_{1}\right)}{\sqrt{2} \epsilon}\right)$. A capillary boundary condition with $\theta=$ $\frac{\pi}{4}$ is imposed for the bottom and top boundaries since $\Gamma_{0}$ intersects these at angle $\frac{\pi}{4}$. Zero Neumann condition is applied on the left and right boundaries since $\Gamma_{0}$ intersects these boundaries orthogonally.

With and without the stochastic component in (5.5), the +1 phase always separates, even when $U_{(0,0)}^{0}$ is perturbed from 0.0 to 0.1 , see Fig. 4 . Similar qualitative pictures were observed when a discontinuous datum was used instead of a continuous one. 

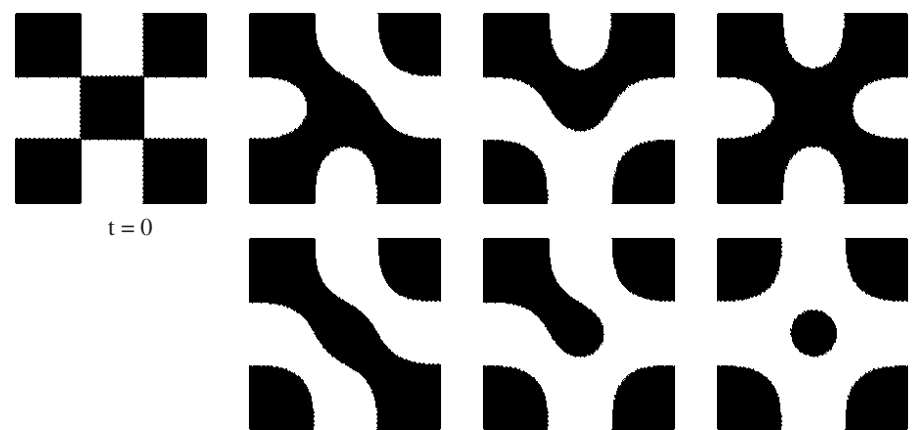

FIG. 5. Example 5.5. Initial datum and six examples from all 16 different possible random evolutions at $t=0.2396$. Black denotes regions where $U_{x}^{k}>0$.

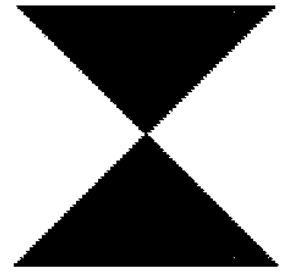

$t=0$

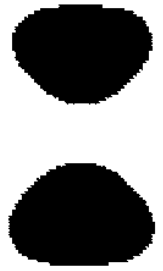

$\mathrm{t}=0.0959$

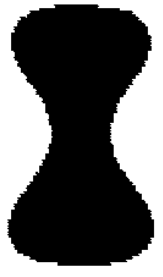

$\mathrm{t}=0.0959$

FIG. 6. Example 5.6. Initial datum and two equi-probable noisy evolutions. Black denotes regions where $U_{x}^{k}>0$.

EXAMPLE $5.5 \Gamma_{0}$ is a tic-tac-toe figure.

The lattice is $150 \times 150$ points centred at $(2.32,2.32)$ on the $r_{1} r_{2}$ plane with initial datum $U_{x}^{0}=\tanh \left(\frac{\left(x_{1}-1.55\right)\left(x_{2}-1.55\right)\left(x_{1}-3.10\right)\left(x_{2}-3.10\right)}{\sqrt{2} \epsilon}\right)$ and zero Neumann boundary conditions. This is a variation of Example 5.1 with now four, instead of one, orthogonal self-intersection points.

In the absence of randomness in (5.5), all +1 phase squares separate to form four shrinking +1 phase quarter-circles and one shrinking +1 phase circle on a background of -1 phase. Preceding examples suggest $2^{4}$ different possible outcomes from this datum when noise is present in the algorithm (5.5). The exponent four corresponds to the number of $\frac{\pi}{2}$ self-intersections on $\Gamma_{0}$. In the presence of randomness, all 16 different configurations were recorded in a batch of 40 experiments. Figure 5 displays six of these different geometric realizations. The same qualitative pictures were observed when a discontinuous datum was used instead of a continuous one.

EXAMPLE 5.6 A figure eight consisting of two triangles which self-intersect at angle $\frac{\pi}{2}$.

The lattice consists of $200 \times 150$ points centred at $(0,0)$ on the $r_{1} r_{2}$ plane with zero Neumann boundary conditions.

When the random term is absent from (5.5), the +1 phase separates. When $U_{(0,0)}^{0}$ is perturbed from 0.0 to +0.05 , the +1 phase joins. In the presence of randomness, the two outcomes (A) and (B) are recorded with almost 0.5 probability. These probabilities appear to be unaffected by the above perturbation, see Figure 6 . The same qualitative pictures were observed when a discontinuous datum was used instead of a continuous one.

EXAMPLE 5.7 A figure eight consisting of two circles of radius 1. 

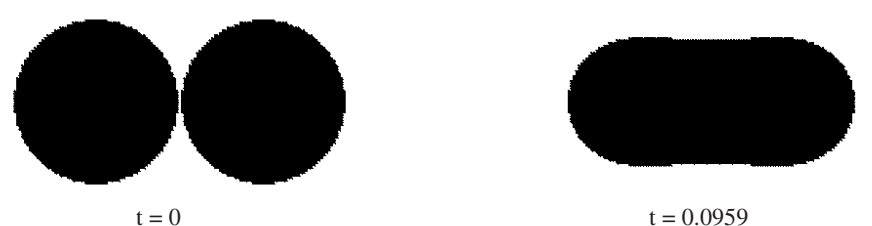

FIG. 7. Example 5.7. Initial datum and the only possible random evolution. Black denotes regions where $U_{x}^{k}>0$.

The initial datum is $U_{x}^{0}=\tanh \left(\frac{\left(1-\left(x_{1}-\rho\right)^{2}-x_{2}^{2}\right)\left(\left(x_{1}+\rho\right)^{2}+x_{2}^{2}-1\right)}{\sqrt{2} \epsilon}\right)$ where the respective circle centres are $( \pm \rho, 0)$. The circles touch at $(0,0)$ in $\mathbb{R}^{2}$ when $\rho=1$. On the computational domain $\mathcal{O}_{\Delta x}$, these circles might not meet precisely at $(0,0)$ for an arbitrary $\Delta x$. We will vary the distance between the circles, $2 \rho$ as defined on this lattice within the interval $[2,2+\delta]$ for an appropriate $0<\delta \ll 1$ and record the corresponding qualitative change in $\Gamma_{k \Delta t}$ for this $\Gamma_{0}$.

The lattice consists of $150 \times 100$ points with zero Neumann boundary conditions. With and without the stochastic component in (5.5), the +1 phase always joins to form a dumbbell-like outline when the distance between the centres of the initial circles was varied on the interval [2, 2.198 181]. Figure 7 shows the noisy evolution when the distance between the centres is 2.198181 . Neither the use of discontinuous datum nor the presence of one-point perturbation changed this result qualitatively.

EXAmPLE 5.8 An example of Belletini \& Paolini with a driving force $(1-t)$.

In [6], the authors construct an example of interface fattening for the forced mean curvature flow

$$
V=-\mu \sigma \kappa+(1-t), \quad(x, t) \in \mathbb{R}^{2} \times\{0 \geqslant t \geqslant 1\} .
$$

Specifically, their example involves two circles of a common initial radius $R_{0}>2$ whose centres are at $\left( \pm R^{*}, 0\right), R^{*}>R_{0}$, such that if $R(t)$ is the radius of any one of the circles at time $t$, then $R(t)$ has a strict maximum at time $t^{*} \in(0,1)$ where $R\left(t^{*}\right)=R^{*}$. It was shown in [6] that the zero level set in the level set formulation fattened after time $t^{*}$ when the circles touch at $(0,0)$. Considered separately, each circle of initial radius $R_{0}$ will expand until it touches $(0,0)$ at time $t^{*}$ when it has achieved maximal radius $R^{*}$, and shrinks for $t>t^{*}$.

Referring to Section 3, the corresponding deterministic Allen-Cahn equation for law (5.7) is easily seen to be (2.11) with the potential $F^{\prime}(u)$ replaced by $F_{u}^{\epsilon}(u ; t)=u\left(u^{2}-1\right)-\epsilon \frac{\sqrt{2}}{3}(1-t)$. The associated SGL equation is (3.2) with this modification. By the techniques in Section 3, this model can be formally shown to approximate the law

$$
V=-\mu \sigma \kappa+(1-t)+\sqrt{\frac{2 \mu \epsilon}{\beta}} \mathcal{J},
$$

for $\epsilon \ll 1$. The SGL scheme used here is again (5.5) with the modified potential. A zero Neumann boundary condition is applied on the computational domain.

For this experiment, we set $R_{0}=3.1$. Using a first-order Euler method with time step size $10^{-4}$ for the differential equation $\dot{R}=R^{-1}+(1-t), R(0)=R_{0}$, we computed $R^{*}$ to be 3.340105 and 


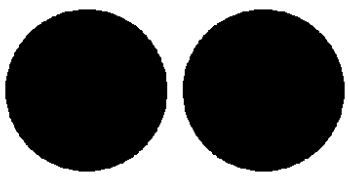

$\mathrm{t}=0$

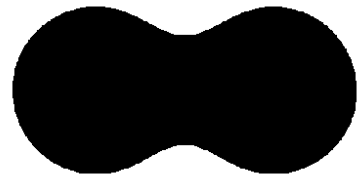

$\mathrm{t}=1.1982$

FIG. 8. Example 5.8. Initial datum and the only possible random evolution. Black denotes regions where $U_{x}^{k}>0$.

$t^{*}$ to be all time values in the time interval [0.6996, 0.7015]. Note that the approximated maximum $R^{*}$ of $R(t)$ occurs not just at one time instant but over a time window due to numerical errors. The initial datum $U_{x}^{0}=\tanh \left(\frac{\left(R_{0}^{2}-\left(x_{1}-\rho\right)^{2}-x_{2}^{2}\right)\left(\left(x_{1}+\rho\right)^{2}+x_{2}^{2}-R_{0}^{2}\right)}{\sqrt{2} \epsilon}\right)$ was used, where $2 \rho$ denotes the distance between the circle centres. As in Example 5.7, we vary this distance within the interval $[2 \times 3.340105,2 \times 3.340105+\delta], \delta>0$, and record the corresponding qualitative change in $\Gamma_{k} \Delta t$ for this $\Gamma_{0}$. We are interested in the subsequent evolution after the circles touch.

With and without the stochastic component in (5.5), we find that almost surely, the +1 phase joins after the circles touch to form a dumbbell-like outline when $2 \rho$ is taken in the interval [6.687 425, 6.935 108]. Fig. 8 shows the noisy evolution when the distance between the centres is 6.811267 .

Concluding remarks for Section 5. When the stochastic component is absent from the SGL scheme (5.4), we have a deterministic phase field approximation which is very sensitive to small perturbations in the initial datum. For instance, in Example 5.1 the subsequent evolution of the cross-shaped $\Gamma_{0}$ that was suspended indefinitely led to $\Gamma_{k} \Delta t$ in the shape of a pair of unconnected hyperbola-like curves, when the $U_{x}^{0}$ is perturbed slightly at a point. From a computational perspective, state suspension and extreme sensitivity to small perturbations in the initial datum are undesirable. This sensitivity to small perturbations in the datum is consistently observed when the deterministic version of (5.4) is applied on curves which self-intersect at angle $\frac{\pi}{2}$. Such an effect is also expected by the results on interface fattening outlined in Section 2.

When random fluctuations are present in (5.4), state suspension is never detected. With curves that self-intersect orthogonally, two qualitatively different outcomes were observed in the subsequent $\Gamma_{k \Delta t}$ evolution with almost equal probability 0.5 : either the +1 phase joins and thus the zero level set of the approximating order parameter, $\Gamma_{k} \Delta t$, looks like a pair of hyperbola-like curves, or the +1 phase separates and $\Gamma_{k \Delta t}$ also looks like another pair of hyperbola-like curves oriented towards a different direction. These simulations suggest that noise picks two of all possible Brakke solutions with equal probability. With the $\Gamma_{0}$ of Example 5.5 which has four orthogonal selfintersections, it is a reasonable conjecture that each of the 16 qualitatively different outcomes have equal probability $\frac{1}{16}$. Small perturbations of the initial data do not appear to affect the probabilities of the outcomes in our experiments.

When scheme (5.4) was used in Examples 5.7 and 5.8 where two circles intersect, the subsequent evolution yields $\Gamma_{k \Delta t}$ as a simply connected curve which looks like the outline of a dumbbell. This same topological picture is obtained when the random term of (5.4) is removed, and when small perturbations are applied to the initial datum. Examples 5.7 and 5.8 are essentially two curves which self-intersect at $\pi$. Noise appears to select the stable outer Brakke envelope almost surely in these 
cases. However, in Example 5.4 where two curves self-intersect at angle $\frac{\pi}{4}$, the subsequent $\Gamma_{k} \Delta t$ looks like a pair of hyperbola-like curves which point in the direction of the $\frac{\pi}{4}$ angle. With and without the random component in (5.4), only one solution seems to be selected every time.

In summary, random fluctuations:

- render the scheme (5.4) robust to small perturbations in the initial datum while the corresponding deterministic scheme is not robust;

- resolve interface fattening, inducing a probabilistic selection mechanism between all possible Brakke solutions of the macroscopic mean curvature flow;

- preserve the probabilities associated with the selected Brakke solutions under small perturbations of the initial datum.

One fundamental conjecture suggested by these simulations is that while uniqueness fails for deterministic mean curvature flow (2.13) or (5.7), it might possibly hold in probability for its stochastic counterpart, (5.2) or (5.8).

\section{Numerical experiments using Ising spin models}

Here, the effects of random fluctuations on geometric flows will be studied employing an entirely different type of microscopic algorithm, namely the Ising spin model with non-conservative dynamics. We will also discuss such effects to the Mullins-Sekerka flow using as an approximating microscopic algorithm, a conservative Ising system with Metropolis dynamics.

\subsection{Non-conservative dynamics}

In this discussion, we refer to Section 2 for relevant equations and notations for an Ising model with spin flip dynamics. Let $\Delta x$ denote the space grid size between adjacent lattice sites and we denote the lattice by $\Lambda$. In practice, we take the radially symmetric interaction potential $J: \mathbb{R}^{2} \rightarrow \mathbb{R}$ to be an indicator function on an octagonal interaction neighbourhood of 37 sites with Metropolis dynamics $\Psi(s)=\exp \left(-s^{+}\right), s \in \mathbb{R}$. The time step size $\Delta t$ is set equal to $(\Delta x)^{2}$ and the probability that the spin at site $x \in \Lambda, \sigma(x)$, switches its sign at a particular iteration is given by

$$
c_{\gamma}(x, \sigma) \Delta t=\Psi\left(\beta 2 \sigma(x) \sum_{z \neq x} J_{\gamma}(x, z) \sigma(z)\right) \Delta t,
$$

where $\gamma^{-1}>0$ is the interaction range. The external field $h$ is set to zero and a zero Neumann boundary condition is used. The macroscopic limit of this model and its associated local mean field equation, (2.5), has been shown in [24] and [25] to be mean curvature motion (2.13).

The experiments here used the initial datum $\Gamma_{0}$ of Example 5.1, i.e. the union of the coordinate axes in $\mathbb{R}^{2}$, variously translated. We set $\Delta x=0.1, \beta=20$ and the lattice size is $100 \times 100$ points. The model at hand is fundamentally different from the SGL scheme of Section 5; thus temperature does not scale in the same way. In our simulations with these data it appears that, again with almost equal probability 0.5 , the +1 phase joined or separated independently of the translation of $\Gamma_{0}$ : see Fig. 9. 

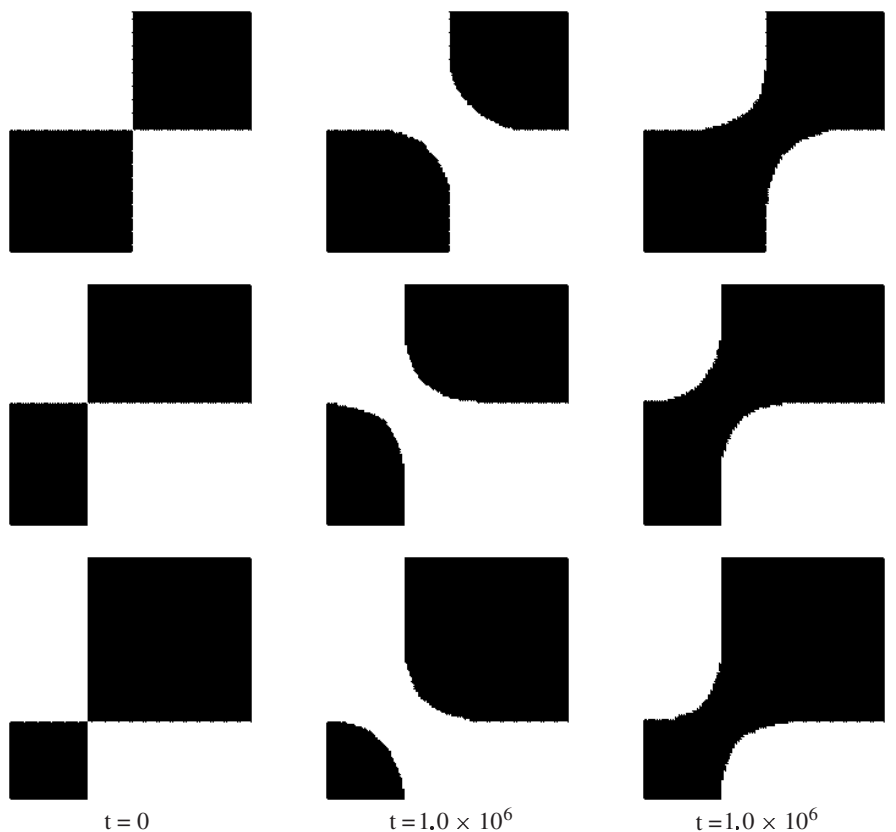

FIG. 9. Section 6.1. Ising model with non-conservative dynamics. Column 1: initial datum of volume fractions $0.5,0.5,0.58$ Columns 2, 3: all possible corresponding two equi-probable random evolutions. Black denotes regions where $U_{x}^{k}>0$.

\subsection{Conservative dynamics}

In this concluding section, we consider an Ising system with only spin exchange dynamics. The equations and notations from Section 2 apply here where we replace $\sigma^{x}$ with $\sigma^{x, y}$ to indicate spin exchange: for any fixed pair $x, y \in \Lambda$ and any configuration $\sigma$,

$$
\sigma^{x, y}(z) \doteq \begin{cases}\sigma(z) & \text { if } z \neq x \text { or } y \\ \sigma(x) & \text { if } z=y \\ \sigma(y) & \text { if } z=x .\end{cases}
$$

Exchanges occur only between neighbouring pairs $x, y \in \Lambda$ of opposite spins. The probability that any such pair exchange spins at a particular iteration is $c_{\gamma}(x, y, \sigma) \Delta t$ where

$$
c_{\gamma}(x, y, \sigma)=\Psi\left(-\beta(\sigma(x)-\sigma(y)) \sum_{z \neq x, y}\left(J_{\gamma}(y, z)-J_{\gamma}(x, z)\right) \sigma(z)\right)
$$

is the spin exchange rate and $\Psi$ is given by the Metropolis dynamics. The external field $h$ is set to zero and a zero Neumann boundary condition is used. In this model, the order parameter is conserved, i.e. the total number of sites with +1 spin is constant in time. Giacomin and Lebowitz [20] showed that as $\gamma \downarrow 0$, the average magnetization $m^{\gamma}(r, t)=\mathrm{E} \sigma_{\gamma^{-2} t}(\gamma x), r=$ $\gamma x \in \mathbb{R}^{d}$, satisfies a mesoscopic diffusion-type equation,

$$
m_{t}=\nabla \cdot\left(\nabla m-\beta m(1-m) \int_{\Omega} \nabla J\left(r-r^{\prime}\right) m\left(r^{\prime}, t\right) \mathrm{d} r^{\prime}\right),
$$



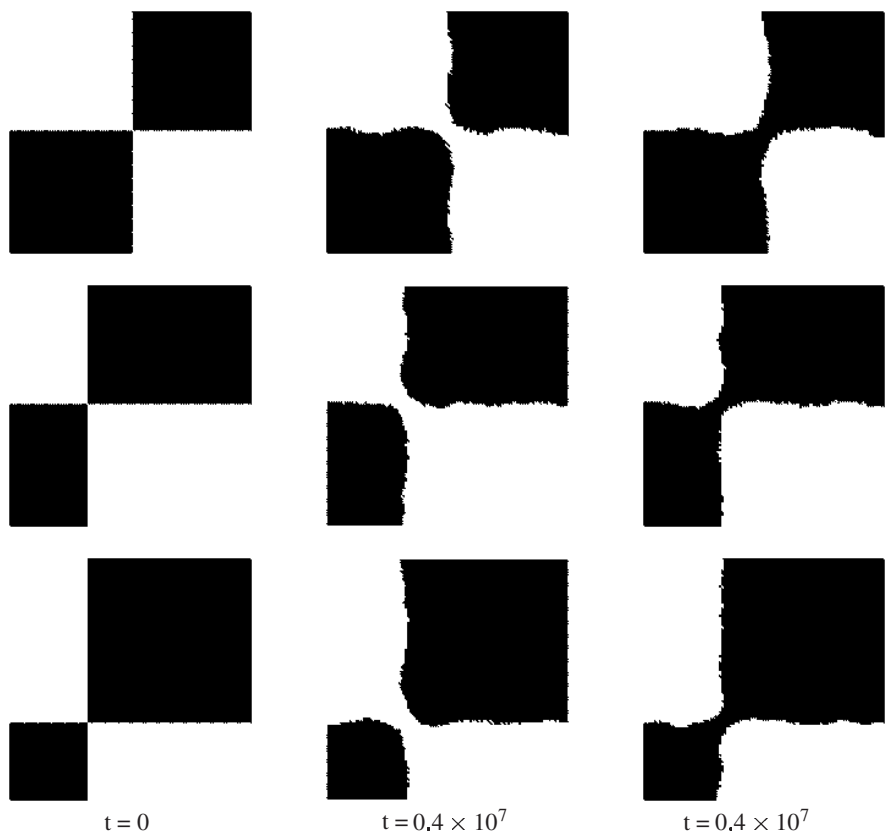

FIG. 10. Section 6.2. Ising model with conservative dynamics. Column 1: initial datum of volume fractions $0.5,0.5,0.58$; Columns 2, 3: all possible corresponding two equi-probable random evolutions. Black denotes regions where $U_{x}^{k}>0$.

which is reminiscent of the Cahn-Hilliard equation. In [21], it was formally shown that (6.3) converges to the volume-preserving Mullins-Sekerka flow under a suitable space-time rescaling, i.e. the interface propagates with a normal velocity

$$
V=\beta[n \cdot \nabla v]_{-}^{+}, \quad(r, t) \in \mathbb{R}^{d} \times\{t \geqslant 0\},
$$

where $n$ is its outward unit normal vector, $[n \cdot \nabla v]_{-}^{+}$denotes the jump of the normal derivative of $v$ across $\Gamma_{t}$ and $v$ solves

$$
\begin{cases}\Delta v=0, & r \in \mathbb{R}^{d} \backslash \Gamma_{t}, \\ v(r, t)=-\sigma \kappa, & r \in \Gamma_{t},\end{cases}
$$

with $\kappa$ being the local mean curvature of $\Gamma_{t}$ and $\sigma$ the surface tension. The analysis in [20] and [21] combined indicates that the Mullins-Sekerka problem (6.4) is the formal macroscopic limit of this conservative Ising system similarly to the mean curvature flow (2.13) being the macroscopic limit of the non-conservative Ising system in the preceding section.

Experiments were performed using the initial datum $\Gamma_{0}$ of Example 5.1 variously translated as in Section 6.1 with the conservative Ising system. We set $\Delta x=0.1, \beta=20$ and the lattice size is $100 \times 100$ points. As with the non-conservative Ising experiments, the simulations here suggest that with almost equal probability 0.5 , the +1 phase joined or separated, irrespective of the translation of $\Gamma_{0}$, see Figure 10.

These simulations indicate that for the Mullins-Sekerka problem (6.4), chessboard-type data generate two different possible evolutions as in the mean curvature flow case. Once again, the random mechanism allows the selection of one of the evolutions with equal probabilities. 


\section{Acknowledgements}

The authors' research is partially supported by the National Science Foundation grants DMS9626804 and DMS-9801769.

\section{REFERENCES}

1. Allen, S. M. \& CAHN, J. W. A macroscopic theory for antiphase boundary motion and its application to antiphase domain coarsening. Acta Metall. 27, (1979) 1085-1095.

2. Angenent, S., Chopp, D., \& Ilmanen, T. A computed example of nonuniqueness of mean curvature flow in $\mathbb{R}^{3}$. Commun. Partial Diff. Equ. 20, (1995) 1937-1958.

3. Angenent, S. B. \& Velásquez, J. J. L. Degenerate neckpinches in mean curvature flow. J. Reine Angew. Math. 482, (1997) 15-66.

4. BRAKKE, K. The motion of a surface by its mean curvature, Mathematical Notes. Princeton University Press, Princeton, NJ (1978).

5. Bausch, R., Dohm, V., Janssen, H. K, \& Zia, R. K. P. Critical dynamics of an interface in $1+$ $\epsilon$ dimensions. Phys. Rev. Lett. 47, (1981) 1837-1840.

6. Belletini, G. \& Paolini, M. Two examples of fattening for the curvature flow with a driving force. Atti Accad. Naz. Lincei Cl. Sci. Fis. Mat. Natur. Rend. Lincei (9) Mat. Appl. 5, (1994) 229-236.

7. BARles, G. \& Souganidis, P. E. A new approach to front propagation problems: theory and applications. Arch. Rational Mech. Anal. 141, (1998) 1337-1348.

8. Barles, G., Soner, H. M., \& Souganidis, P. E. Front propagation and phase field theory. SIAM J. Control Optim. 31, (1993) 439-469.

9. Chen, X., Elliott, C. M., Gardiner, A. R., \& Zhao, J. J. Convergence of numerical solutions to the Allen-Cahn equation. Appl. Anal. 69, (1998) 47-56.

10. Chen, Y. G., GigA, Y., \& Goto, S. Uniqueness and existence of viscosity solutions of generalized mean curvature flow equations. J. Differ. Geom. 33, (1991) 749-786.

11. Crandall, M. G., Ishit, H., \& Lions, J. P. User's guide to viscosity solutions of second order partial differential equations. Bull. AMS 27, (1992) 1-67.

12. De Masi, A., Orlandi, E., Presutti, E., \& Triolo, L. Glauber evolution with the Kac potentials I. Mesoscopic and macroscopic limits, interface dynamics. Nonlinearity 7, (1994) 633-696.

13. De Masi, A., Orlandi, E., Presutti, E., \& Triolo, L. Glauber evolution with Kac potentials III: spinodal decomposition. Nonlinearity 9, (1996) 53-114.

14. Elliott, C. M. Approximation of curvature dependent interface motion. State of the Art in Numerical Analysis. Clarendon, Oxford (1997) pp. 407-440.

15. Evans, L. C. \& SprucK, J. Motion of level sets by mean curvature I. J. Differ. Geom. 33, (1991) 635-681.

16. Evans, L. C., Soner, H. M., \& Souganidis, P. E. Phase transitions and generalized motion by mean curvature. Commun. Pure Appl. Math. 45, (1992) 1097-1123.

17. FUNAKI, T. Singular limit for stochastic reaction-diffusion equation and generation of random interfaces. Acta Math. Sin. (Engl. Ser.) 15, (1999) 407-438.

18. GARDiner, C. W. Handbook of Stochastic Methods for Physics, Chemistry and the Natural Sciences (Springer Series in Synergetics). Springer, Berlin (1985).

19. Gurtin, M. Thermomechanics of evolving phase boundaries in the plane. Oxford Mathematical Monographs. Oxford University Press, Oxford (1993).

20. Giacomin, G. \& Lebowitz, J. L. Phase segregation dynamics in particle systems with long range interactions. I. Macroscopic limits. J. Stat. Phys. 87, (1997) 37-61.

21. Giacomin, G. \& Lebowitz, J. L. Phase segregation dynamics in particle systems with long range interactions II: interface motion. SIAM J. Appl. Math. 58, (1998) 1707-1729. 
22. Ilmanen, T. Convergence of the Allen-Cahn equation to Brakke's motion by mean curvature. J. Differ. Geom. 38, (1993) 417-461.

23. Katsoulakis, M. \& Souganidis, P. E. Interacting particle systems and Generalized evolution of fronts. Arch. Rat. Mech. Anal. 127, (1994) 133-157.

24. Katsoulakis, M. \& Souganidis, P. E. Generalized motion by mean curvature as a macroscopic limit of stochastic Ising models with long range interactions and Glauber dynamics. Commun. Math. Phys. 169, (1995) 61-97.

25. Katsoulakis, M. \& Souganidis, P. E. Stochastic Ising models and anisotropic front propagation. $J$. Stat. Phys. 87, (1997) 63-89.

26. Katsoulakis, M. \& Vlachos, D. From microscopic interactions to macroscopic laws of cluster evolution. Phys. Rev. Lett. 84, (2000) 1511-1514.

27. KAWASAKI, K. \& OHTA, T. Kinetic drumhead model of interface I. Prog. Theor. Phys. 67, (1982) 147163.

28. Kloeden, P. E. \& Platen, E. Numerical solution of stochastic differential equations. Applications of Mathematics, vol. 23. Springer, Berlin (1992).

29. LiOnS, P. L. \& SougANidis, P. E. Fully nonlinear stochastic partial differential equations: non-smooth equations and applications. C. R. Acad. Sci. Paris Series I Math. 327, (1998) 735-741.

30. Osher, S. \& Sethian, J. Fronts propagating with curvature-dependent speed: algorithms based on Hamilton-Jacobi formulations. J. Comput. Phys. 79, (1988) 12-49.

31. Rubinstein, J., Sternberg, P., \& Keller, J. B. Fast reaction, slow diffusion, and curve shortening. SIAM J. Appl. Math. 49, (1989) 116-133.

32. SpoHn, H. Interface motion in models with stochastic dynamics. J. Stat. Phys. 71, (1993) 1081-1132.

33. Sethian, J. Level set methods: evolving interfaces in geometry, fluid mechanics, computer vision, and materials science. Cambridge Monographs on Applied and Computational Mathematics. Cambridge University Press, Cambridge (1996).

34. Walsh, J. B. An introduction to stochastic partial differential equations (Ecole d'été de probabilites de Saint Flour XIV, 1984). Lecture Notes in Mathematics. Springer, Berlin (1986).

35. YIP, N. K. Stochastic motion by mean curvature. Arch. Rat. Mech. Anal. 144, (1998) 313-355.

Appendix $\ell^{2}$-stability of the fully-discrete SGL scheme (5.4)

THEOREM 5.1 Suppose that $F^{\prime}$ is globally Lipschitz continuous, and $U=\bar{U}$ on $\partial \mathcal{O}_{\Delta x} \times \Sigma_{\Delta t}$. Then, there exists a constant $C=C(F, \epsilon)>0$ such that $\sup _{0 \leqslant k \leqslant N} \mathrm{E}\left\|U^{k}-\bar{U}^{k}\right\|_{\ell^{2}} \leqslant C \mathrm{E} \| U^{0}-$ $\bar{U}^{0} \|_{\ell^{2}}$.

Proof. First, we will show the $\ell^{2}$-stability of the semi-discrete scheme (5.3). Suppose that $F^{\prime}(\cdot)$ has a Lipschitz constant $A$. Let $u(x, t)$ and $\bar{u}(x, t)$ solve (5.3) for initial data $u_{0}(x)$ and $\bar{u}_{0}(x)$ respectively where $(x, t) \in \mathcal{O}_{\Delta x} \times\{t>0\}$. Define $v(x, t) \doteq u(x, t)-\bar{u}(x, t)$ which solves $\epsilon^{2} \frac{\mathrm{d}}{\mathrm{d} t} v(x, t)=\epsilon^{2} \Delta_{\Delta x} v(x, t)-\left(F^{\prime}(u(x, t))-F^{\prime}(\bar{u}(x, t))\right)$. The noise term vanishes because $u$ and $\bar{u}$ solve the scheme for the same stochastic term. Using the Lipschitz property we get

$$
\begin{aligned}
\frac{1}{2} \frac{\mathrm{d}}{\mathrm{d} t}|v(x, t)|^{2} & \leqslant \frac{\sum_{j=1}^{d} v\left(x+\Delta x e_{j}, t\right)-2 v(x, t)+v\left(x-\Delta x e_{j}, t\right)}{\Delta x^{2}} v(x, t)+\frac{A}{\epsilon^{2}}|v(x, t)|^{2} \\
& =\frac{\sum_{j=1}^{d} v\left(x+\Delta x e_{j}, t\right) v(x, t)+v\left(x-\Delta x e_{j}, t\right) v(x, t)}{\Delta x^{2}}+\left(\frac{A}{\epsilon^{2}}-\frac{2 d}{\Delta x^{2}}\right)|v(x, t)|^{2},
\end{aligned}
$$




$$
\begin{aligned}
\frac{1}{2} \frac{\mathrm{d}}{\mathrm{d} t} \sum_{x \in \mathcal{O}_{\Delta x}}|v(x, t)|^{2} \leqslant & \sum_{x \in \mathcal{O}_{\Delta x}}\left(\frac{\sum_{j=1}^{d}\left|v\left(x+\Delta x e_{j}, t\right)\right|^{2}+2|v(x, t)|^{2}+\left|v\left(x-\Delta x e_{j}, t\right)\right|^{2}}{2 \Delta x^{2}}\right) \\
& +\left(\frac{A}{\epsilon^{2}}-\frac{2 d}{\Delta x^{2}}\right) \sum_{x \in \mathcal{O}_{\Delta x}}|v(x, t)|^{2} \\
\leqslant & \sum_{x \in \partial \mathcal{O}_{\Delta x}} \frac{|v(x, t)|^{2}}{2 \Delta x^{2}}+\frac{2 d}{\Delta x^{2}}\|v(x, t)\|_{\ell^{2}}^{2} \\
& +\left(\frac{A}{\epsilon^{2}}-\frac{2 d}{\Delta x^{2}}\right)\|v(x, t)\|_{\ell^{2}}^{2} \\
\leqslant & \frac{1}{2} \sum_{x \in \partial \mathcal{O}_{\Delta x}}\left(\frac{v(x, t)}{\Delta x}\right)^{2}+\frac{A}{\epsilon^{2}}\|v(x, t)\|_{\ell^{2}}^{2} .
\end{aligned}
$$

By Gronwall's inequality and assuming that $v(x, t)=0$ on $x \in \partial \mathcal{O}_{\Delta x}$,

$$
\begin{aligned}
\|v(x, t)\|_{\ell^{2}}^{2} & \leqslant \mathrm{e}^{\frac{2 A t}{\epsilon^{2}}}\left\{\|v(x, 0)\|_{\ell^{2}}^{2}+\int_{0}^{t} \sum_{x \in \partial \mathcal{O}_{\Delta x}}\left(\frac{v(x, t)}{\Delta x}\right)^{2} \mathrm{~d} s\right\} \\
\mathrm{E}\|v(x, t)\|_{\ell^{2}}^{2} & \leqslant \mathrm{e}^{\frac{2 A t}{\epsilon^{2}}} \mathrm{E}\|v(x, 0)\|_{\ell^{2}}^{2} .
\end{aligned}
$$

Next, we demonstrate the $\ell^{2}$-stability for the fully discrete scheme (5.4), guided by calculations for the semi-discrete scheme above. Let $V_{x}^{k}=U_{x}^{k}-\bar{U}_{x}^{k}$ which solves $V_{x}^{k+1}=(1-2 d \mu) V_{x}^{k}+$ $\mu \sum_{j=1}^{d}\left(V_{x-\Delta x e_{j}}^{k}+V_{x+\Delta x e_{j}}^{k}\right)-\Delta t\left(F^{\prime}\left(U_{x}^{k}\right)-F^{\prime}\left(\bar{U}_{x}^{k}\right)\right)$. Again, the noise term disappears since the same noise is used in computing both $U_{x}^{k}$ and $\bar{U}_{x}^{k}$. Without loss of generality, say that $V_{x}^{k}>0$ and let each $A^{\epsilon}$ denote $\frac{A}{\epsilon^{2}}$ to avoid the unnecessary clutter of modulus symbols and $\epsilon$. Suppose that $1-2 d \mu>0$ then

$$
\begin{aligned}
\left|V_{x}^{k+1}\right|^{2}= & (1-2 d \mu) V_{x}^{k} V_{x}^{k+1}+\mu \sum_{j=1}^{d}\left(V_{x-\Delta x e_{j}}^{k}+V_{x+\Delta x e_{j}}^{k}\right) V_{x}^{k+1} \\
& -\Delta t\left(F^{\prime}\left(U_{x}^{k}\right)-F^{\prime}\left(\bar{U}_{x}^{k}\right)\right) \underline{V_{x}^{k+1}} \\
\leqslant & \underbrace{(1-2 d \mu) V_{x}^{k} V_{x}^{k+1}}_{\mathrm{I}}+\underbrace{\mu \sum_{j=1}^{d}\left(V_{x-\Delta x e_{j}}^{k}+V_{x+\Delta x e_{j}}^{k}\right) V_{x}^{k+1}}_{\mathrm{II}} \\
& \begin{array}{l}
+\Delta t A^{\epsilon}\left(1-2 d \mu+\Delta t A^{\epsilon}\right)\left|V_{x}^{k}\right|^{2} \\
+\Delta t A^{\epsilon} \mu \sum_{j=1}^{d}\left(V_{x-\Delta x e_{j}}^{k}+V_{x+\Delta x e_{j}}^{k}\right) V_{x}^{k} .
\end{array}
\end{aligned}
$$

We sum the expression above over $x \in \mathcal{O}_{\Delta x}$. Using Jensen's inequality, the summed terms I, II and 
III are easily seen to be bounded by

$$
\begin{aligned}
& \text { I : } \quad(1-2 d \mu)\left(1+\Delta t A^{\epsilon}\right)\left\|V^{k}\right\|_{\ell^{2}}^{2}+\frac{\mu}{2} \sum_{x \in \partial \mathcal{O}_{\Delta x}}\left|V_{x}^{k}\right|^{2}, \\
& \text { II : } 2 d \mu\left(1+\Delta t A^{\epsilon}\right)\left\|V^{k}\right\|_{\ell^{2}}^{2}+\mu \frac{1+2 d \mu+\Delta t A^{\epsilon}}{2} \sum_{x \in \partial \mathcal{O}_{\Delta x}}\left|V_{x}^{k}\right|^{2}, \\
& \text { III : } \Delta t A^{\epsilon}\left(1+\Delta t A^{\epsilon}\right)\left\|V^{k}\right\|_{\ell^{2}}^{2}+\frac{\Delta t A^{\epsilon} \mu}{2} \sum_{x \in \partial \mathcal{O}_{\Delta x}}\left|V_{x}^{k}\right|^{2} .
\end{aligned}
$$

Putting terms I, II and III together yields the $\ell^{2}$ bound on $V^{k+1}$,

$$
\begin{aligned}
&\left\|V^{k+1}\right\|_{\ell^{2}}^{2} \leqslant\left(1+\frac{\Delta t A}{\epsilon^{2}}\right)^{2}\left\|V^{k}\right\|_{\ell^{2}}^{2}+\mu\left(1+\frac{\Delta t A}{\epsilon^{2}}\right) \sum_{x \in \partial \mathcal{O}_{\Delta x}}\left|V_{x}^{k}\right|^{2} \\
& \mathrm{E}\left\|V^{k+1}\right\|_{\ell^{2}}^{2} \leqslant \mathrm{e}^{\frac{2(k+1) \Delta t A}{\epsilon^{2}}} \mathrm{E}\left\|V^{0}\right\|_{\ell^{2}}^{2} .
\end{aligned}
$$

Assuming that that on $(x, i) \in \partial \mathcal{O}_{\Delta x} \times\{0,1, \ldots, N\}, V_{x}^{i}=0$, we have the final line above. 